\title{
Reactions of endo-3-Diazotricyclo[3.2.1.0 $\left.0^{2,4}\right]$ oct-6-ene, a
}

\section{Potential Precursor for the Generation of a Neutral $\mathrm{C}_{8} \mathrm{H}_{8}$}

\section{Molecule with a Pyramidally Coordinated Carbon}

\author{
Jean-Luc Mieusset and Udo H. Brinker
}

Institut für Organische Chemie, Universität Wien, Währinger Straße 38, A-1090 Wien, Austria

E-mail: Udo.Brinker@univie.ac.at

Corresponding author. Phone: +43-1-4277-52121. Fax: +43-1-4277-52140

\section{Supplementary Information}

Cartesian coordinates and thermodynamic data for all relevant stationary points (B3LYP/6$311+\mathrm{G}(\mathrm{d}, \mathrm{p})$ geometries $)$ :

Neutral compounds

2

Cationic compounds 


\section{Tetracyclo[3.2.0.0 $\left.{ }^{1,3} \cdot 0^{1,6}\right]$ heptane (2), NImag $=0$}

$1 \backslash 1 \backslash G I N C-N O D E 128 \backslash$ Freq $\backslash R B 3 L Y P \backslash 6-311+G(d, p) \backslash C 7 H 8 \backslash M I E U S S E T \backslash 03-M a r-2006 \backslash 0 \backslash$ $\backslash \# N$ GEOM=ALLCHECK GUESS=READ SCRF=CHECK GENCHK RB3LYP/6-311+G(D,P) FRE

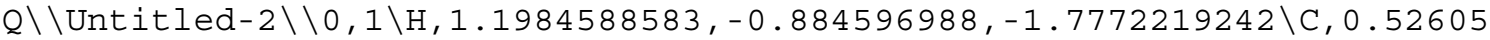
$62879,-0.3664166431,-1.1075816733 \backslash C, 0.0025636131,1.5018974886,0.494656$ $6922 \backslash C,-0.8507240565,-0.8608197391,0.4208019024 \backslash C,-0.3696496855,0.2212$ $364209,1.2067434859 \backslash \mathrm{C},-0.9572796313,-0.6570291926,-1.0734599336 \backslash \mathrm{C}, 1.00$ $89952214,0.7198333825,-0.3185939139 \backslash \mathrm{H}, 0.4942058391,2.2021445593,1.1686$ $886407 \backslash \mathrm{H},-0.3548794108,0.1385502422,2.2846578 \backslash \mathrm{H},-1.6352744427,0.126327$ $8178,-1.4293574743 \backslash \mathrm{H}, 2.045075449,1.0196547292,-0.3940861265 \backslash \mathrm{H},-0.79058$ $31593,2.0262490391,-0.0493670927 \backslash \mathrm{H},-1.2002456406,-1.7628891017,0.90356$ $4744 \backslash \mathrm{H},-1.1915292161,-1.5894937203,-1.585333104 \backslash \mathrm{C}, 0.8791668715,-0.7713$ $594802,0.5238425298 \backslash \backslash$ Version=AM64L-G03RevC.02 \State $=1-A^{\prime} \backslash H F=-271.42089$ $14 \backslash \mathrm{RMSD}=1.889 \mathrm{e}-09 \backslash \mathrm{RMSF}=8.200 \mathrm{e}-05 \backslash \mathrm{Dipole}=-0.7492143,0.6485974,-0.434372$

Sum of electronic and zero-point Energies= Sum of electronic and thermal Energies= Sum of electronic and thermal Enthalpies= $-271.296037$ $-271.290005$ $-271.289061$ Sum of electronic and thermal Free Energies= $-271.325130$

\section{Pentacyclo[3.3.0.0 $\left.{ }^{2,4} \cdot 0^{2,8} \cdot 0^{3,7}\right]$ octane (4), NImag $=0$}

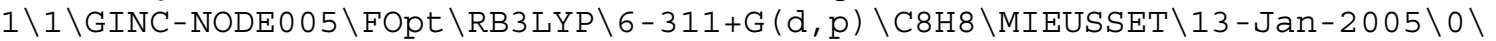

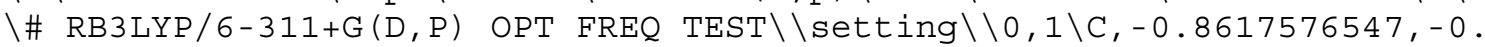
$3029721777,-1.3421599195 \backslash C,-0.433145248,-1.199173473,-0.1636609484 \backslash C_{,}-$ $0.7476799255,-0.3471245997,1.0671682973 \backslash C,-0.5976215037,1.0087467793,0$ $.6654996853 \backslash C,-0.192698028,0.9792739998,-0.8095265489 \backslash C, 1.043220198,-0$ $.8522975151,0.0351356042 \backslash C, 1.1937244761,0.5062253725,-0.3682012127 \backslash C, 0$ $.8360893025,0.2928260208,1.2917083526 \backslash \mathrm{H},-1.9486568676,-0.2073484869,-1$ $.42448675 \backslash \mathrm{H},-0.4393992045,-0.6324055809,-2.2960751612 \backslash \mathrm{H},-0.7258614784$, $-2.2447495203,-0.1593109271 \backslash \mathrm{H},-1.1528177159,-0.6938314512,2.0046957506$ $\backslash \mathrm{H},-0.8722095816,1.8785680603,1.2411654695 \backslash \mathrm{H},-0.2693239025,1.895737228$ $1,-1.3867620357 \backslash \mathrm{H}, 1.841075509,-1.5406925547,0.2654291112 \backslash \mathrm{H}, 2.126403540$ $7,1.031695864,-0.5004353169 \backslash \backslash$ Version $=x 86-$ Linux-G03RevB. 02 $\backslash$ State $=1-A \backslash H F$ $=-309.5226262 \backslash \mathrm{RMSD}=7.190 \mathrm{e}-09 \backslash \mathrm{RMSF}=7.889 \mathrm{e}-05 \backslash \mathrm{Dipole}=-0.6371652,-0.22088$ $55,-0.9743767 \backslash P G=C 01[X(C 8 H 8)] \backslash \backslash @$

Sum of electronic and zero-point Energies=

Sum of electronic and thermal Energies=

Sum of electronic and thermal Enthalpies=

Sum of electronic and thermal Free Energies=

$$
\begin{array}{r}
-309.391661 \\
-309.385687 \\
-309.384743 \\
-309.421184
\end{array}
$$

\section{$(4 \rightarrow 7)$, NImag $=1$}

$1 \backslash 1 \backslash G I N C-N O D E 148 \backslash F T S \backslash R B 3 L Y P \backslash 6-311+G(d, p) \backslash C 8 H 8 \backslash M I E U S S E T \backslash 14-J a n-2005 \backslash 0 \backslash \backslash$

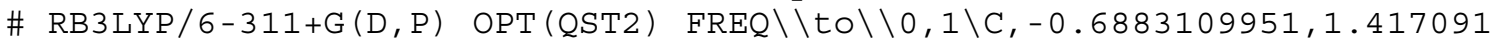
$1121,0.1965959979 \backslash C,-0.3409674666,0.5915898747,-1.0611025142 \backslash C,-0.9894$ $379913,-0.7582732968,-0.7118825013 \backslash C,-0.9118208451,-0.9076174093,0.636$ $8702401 \backslash C,-0.2109549486,0.3415693221,1.1962467981 \backslash C, 1.1103411429,0.159$ $2105904,-0.7934621451 \backslash C, 1.1950934104,-0.0038612584,0.6779441427 \backslash C, 1.03$ $22968068,-1.2936231474,-0.2028164254 \backslash \mathrm{H},-1.7624383863,1.6181789415,0.28$ $07348611 \backslash \mathrm{H},-0.1183844558,2.3462609233,0.2666773426 \backslash \mathrm{H},-0.5667136851,1.0$ $140020905,-2.0367893342 \backslash \mathrm{H},-1.3082829629,-1.5053708881,-1.4243752203 \backslash \mathrm{H}$, $-1.1571060982,-1.7961032218,1.2006927925 \backslash \mathrm{H},-0.3192501359,0.538134136,2$ $.2597290065 \backslash \mathrm{H}, 1.9500717702,0.4030396619,-1.4310931629 \backslash \mathrm{H}, 2.1046692732,0$ $.1053436319,1.254062157 \backslash \backslash$ Version $=x 86-$ Linux-G03RevB. 02 $\backslash$ State $=1-A \backslash H F=-30$ $9.5200089 \backslash \mathrm{RMSD}=3.980 \mathrm{e}-09 \backslash \mathrm{RMSF}=4.806 \mathrm{e}-05 \backslash \mathrm{Dipole}=-0.602093,0.8495769,0.1$ $28849 \backslash \mathrm{PG}=\mathrm{C} 01[\mathrm{X}(\mathrm{C} 8 \mathrm{H} 8)] \backslash \backslash \backslash$

Sum of electronic and zero-point Energies= Sum of electronic and thermal Energies= Sum of electronic and thermal Enthalpies= Sum of electronic and thermal Free Energies=

$$
\begin{array}{r}
-309.389085 \\
-309.383979 \\
-309.383035 \\
-309.417742
\end{array}
$$




\section{$(4 \rightarrow$ 14a), NImag $=1$}

$1 \backslash 1 \backslash G I N C-N O D E 083 \backslash F T S \backslash R B 3 L Y P \backslash 6-311+G(d, p) \backslash C 8 H 8 \backslash M I E U S S E T \backslash 14-J a n-2005 \backslash 0 \backslash \backslash$

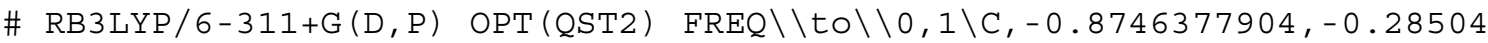
$02334,-1.3455351624 \backslash \mathrm{C},-0.4331636267,-1.1801651488,-0.1719755177 \backslash \mathrm{C},-0.7$ $285154658,-0.3308822146,1.0827925014 \backslash \mathrm{C},-0.5684644916,1.0254587674,0.64$ $88891074 \backslash \mathrm{C},-0.204975563,0.99926113,-0.8218186606 \backslash \mathrm{C}, 1.057369711,-0.8326$ $487412,0.0286935962 \backslash \mathrm{C}, 1.1679865421,0.5382125017,-0.3761515447 \backslash \mathrm{C}, 0.8275$ $070811,0.101990116,1.3499844869 \backslash \mathrm{H},-1.9620764901,-0.1886277522,-1.41909$ $74416 \backslash \mathrm{H},-0.4611752528,-0.6099845186,-2.3050275089 \backslash \mathrm{H},-0.7262629199,-2.2$ $260538444,-0.1705699311 \backslash \mathrm{H},-1.2727287333,-0.6327328505,1.9660162659 \backslash \mathrm{H},-$ $0.7109682835,1.9103991616,1.2476067205 \backslash \mathrm{H},-0.2814355269,1.9211424759,-1$ $.3893375789 \backslash \mathrm{H}, 1.8873095467,-1.5212843272,0.0955408547 \backslash \mathrm{H}, 2.0686992794,1$ $.130024593,-0.3944042196 \backslash \backslash$ Version=x86-Linux-G03RevB. $02 \backslash$ State $=1-A \backslash H F=-3$ $09.5222566 \backslash \mathrm{RMSD}=5.293 e-09 \backslash \mathrm{RMSF}=4.632 e-05 \backslash \mathrm{Dipole}=-0.6270972,-0.0213228$, $-1.0475883 \backslash \mathrm{PG}=\mathrm{C} 01[\mathrm{X}(\mathrm{C} 8 \mathrm{H} 8)] \backslash \backslash @$

Sum of electronic and zero-point Energies= Sum of electronic and thermal Energies= Sum of electronic and thermal Enthalpies=

Sum of electronic and thermal Free Energies=

\author{
$-309.391473$ \\ $-309.386328$ \\ $-309.385384$ \\ $-309.420085$
}

\section{endo-3-Diazotricyclo[3.2.1.0 $\left.{ }^{2,4}\right]$ oct-6-ene (endo-6)}

syn $(\mathrm{NImag}=0)$ :

$1 \backslash 1 \backslash G I N C-N O D E 119 \backslash F r e q \backslash R B 3 L Y P \backslash 6-311+G(d, p) \backslash C 8 H 8 N 2 \backslash M I E U S S E T \backslash 24-J a n-2006 \backslash$

$0 \backslash \backslash \# \mathrm{~N}$ GEOM=ALLCHECK GUESS=READ SCRF=CHECK TEST GENCHK RB3LYP/6-311+G(D

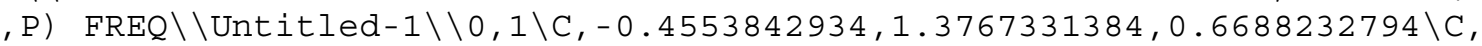
$-0.4554253121,1.3766924437,-0.6689147315 \backslash \mathrm{C},-1.0954362561,0.079090656,1$ $.1290227763 \backslash \mathrm{C},-1.0955093169,0.0790239439,-1.1289965938 \backslash \mathrm{C},-0.1506864581$ $,-1.1289707988,0.7667405626 \backslash \mathrm{C},-2.1532815235,-0.1067102884,0.0000524353$ $\backslash \mathrm{C}, 1.1064666794,-0.9684205663,-0.0000321653 \backslash \mathrm{C},-0.1507378274,-1.1290160$ $929,-0.7667015551 \backslash \mathrm{N}, 2.0767427172,-0.1205450898,-0.0000544839 \backslash \mathrm{N}, 3.02503$ $83666,0.5111817311,0.0000386571 \backslash \mathrm{H}, 0.0007077973,2.1093472155,1.32226464$ $75 \backslash \mathrm{H}, 0.0006243412,2.1092687897,-1.3224281 \backslash \mathrm{H},-1.4479640718,0.0512508742$ , $2.1589451912 \backslash \mathrm{H},-1.4481025661,0.0511211169,-2.1588948828 \backslash \mathrm{H},-0.28835526$ $17,-2.0447385007,1.3346171111 \backslash \mathrm{H},-2.6123412534,-1.0995978374,0.00009623$ $71 \backslash \mathrm{H},-2.9286203132,0.6631780606,0.0000545084 \backslash \mathrm{H},-0.2884504097,-2.044820$ $8221,-1.3345079724 \backslash \backslash$ Version=AM64L-G03RevC.02\State $=1-\mathrm{A} \backslash \mathrm{HF}=-419.1081875$ $\backslash \mathrm{RMSD}=6.792 \mathrm{e}-09 \backslash \mathrm{RMSF}=1.873 e-05 \backslash \mathrm{Dipole}=-1.0535363,-0.1580418,-0.0000078$ Sum of electronic and zero-point Energies= Sum of electronic and thermal Energies= Sum of electronic and thermal Enthalpies= Sum of electronic and thermal Free Energies=

$-418.965032$

$-418.957777$

$-418.956833$

$-418.996882$

TS $($ NImag $=1)$ :

$1 \backslash 1 \backslash$ GINC-NODE062 Freq \RB3LYP \6-311+G(d,p) \C8H8N2 \MIEUSSET $24-J a n-2006 \backslash$ $0 \backslash \backslash \# N$ GEOM=ALLCHECK GUESS=READ SCRF=CHECK TEST GENCHK RB3LYP/6-311+G(D ,P) FREQ $\backslash \backslash$ to $\backslash \backslash 0,1 \backslash \mathrm{C},-0.6570146288,1.4932848598,0.6687298708 \backslash \mathrm{C},-0.65701$ $3619,1.4932440985,-0.6687054982 \backslash C,-1.1876943137,0.1462018416,1.1311950$ $229 \backslash \mathrm{C},-1.1877711063,0.1461632208,-1.1310915192 \backslash \mathrm{C},-0.1282673082,-0.9643$ $746968,0.7741042598 \backslash \mathrm{C},-2.2202188466,-0.134769188,0.0000916256 \backslash \mathrm{C}, 1.0652$ $996594,-0.6224043608,-0.0000349406 \backslash \mathrm{C},-0.1283461532,-0.9644180467,-0.77$ $40298844 \backslash \mathrm{N}, 2.3077780458,-0.3848532226,-0.000106769 \backslash \mathrm{N}, 3.4343656808,-0.1$ $649086475,-0.0001818675 \backslash \mathrm{H},-0.2559822297,2.2574754605,1.3220244915 \backslash \mathrm{H},-0$ $.2559901204,2.2573992375,-1.3220478105 \backslash \mathrm{H},-1.5421238091,0.0894770769,2$. $1594730731 \backslash \mathrm{H},-1.5422680089,0.0894114189,-2.1593450689 \backslash \mathrm{H},-0.1714581537$, $-1.8980383533,1.328883577 \backslash \mathrm{H},-2.5858112391,-1.1660005044,0.0001253032 \backslash \mathrm{H}$ $,-3.0636101016,0.5591543145,0.0001057539 \backslash \mathrm{H},-0.1716045252,-1.89811193,-$ $1.328752484 \backslash \backslash$ Version=AM64L-G03RevC.02 \State $=1-\mathrm{A} \backslash \mathrm{HF}=-419.1054243 \backslash \mathrm{RMSD}=1$ $.954 e-09 \backslash R M S F=1.323 e-05 \backslash D i p o l e=-1.1446439,-0.2602362,0.0000639$ 
Sum of electronic and zero-point Energies= Sum of electronic and thermal Energies= Sum of electronic and thermal Enthalpies= Sum of electronic and thermal Free Energies=
$-418.962592$

$-418.955971$

$-418.955026$

$-418.993691$

anti $(\mathrm{NImag}=0)$ :

$1 \backslash 1 \backslash$ GINC-NODE0 56 Freq $\backslash R B 3 L Y P \backslash 6-311+G(d, p) \backslash C 8 H 8 N 2 \backslash M I E U S S E T \backslash 24-J a n-2006 \backslash$ $0 \backslash \backslash \# N$ GEOM=ALLCHECK GUESS=READ SCRF=CHECK TEST GENCHK RB3LYP/6-311+G (D

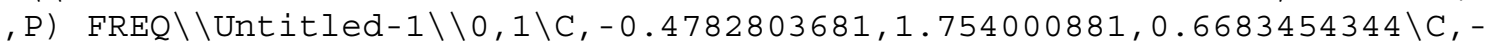
$0.4783212533,1.753960014,-0.6684330422 \backslash C,-1.1311398409,0.4605731504,1$. $1305187113 \backslash \mathrm{C},-1.1312093067,0.4605041078,-1.1304875588 \backslash \mathrm{C},-0.1871013733$, $-0.737721057,0.761891616 \backslash \mathrm{C},-2.1897689392,0.2832351213,0.0000533999 \backslash \mathrm{C}, 1$ $.0700378527,-0.455035317,-0.000025004 \backslash C,-0.1871480496,-0.737767575,-0$. $7618454995 \backslash \mathrm{N}, 2.1597561081,-1.1507631017,-0.000030097 \backslash \mathrm{N}, 3.1893149067,-1$ $.6391556921,-0.0000074954 \backslash \mathrm{H},-0.0023072615,2.4736265838,1.3214007494 \backslash \mathrm{H}$, $-0.0023881596,2.473545779,-1.3215615143 \backslash \mathrm{H},-1.4865115204,0.4353815436,2$ $.1597053824 \backslash \mathrm{H},-1.4866445287,0.4352502764,-2.1596507567 \backslash \mathrm{H},-0.2868052296$ $,-1.6649224706,1.3170290467 \backslash \mathrm{H},-2.6555125525,-0.7068459465,0.0000979864$ $\backslash \mathrm{H},-2.9588523145,1.0579028835,0.0000535313 \backslash \mathrm{H},-0.2868878666,-1.66500304$ $56,-1.3169196212 \backslash \backslash$ Version=AM64L-G03RevC.02 \State $=1-A \backslash H F=-419.1080989 \backslash R$ $\mathrm{MSD}=4.199 \mathrm{e}-09 \backslash \mathrm{RMSF}=1.617 \mathrm{e}-05 \backslash \mathrm{Dipole}=-0.994123,-0.0416156,0.0000189$

Sum of electronic and zero-point Energies= Sum of electronic and thermal Energies= Sum of electronic and thermal Enthalpies= Sum of electronic and thermal Free Energies=

$-418.965143$

$-418.957795$

$-418.956851$

$-418.997166$

\section{$($ endo-6 $\rightarrow$ endo-7), NImag $=1$}

syn:

$1 \backslash 1 \backslash$ GINC-NODE0 56 Freq $\backslash R B 3 L Y P \backslash 6-311+G(d, p) \backslash C 8 H 8 N 2 \backslash M I E U S S E T \backslash 24-J a n-2006 \backslash$ $0 \backslash \backslash \# N$ GEOM=ALLCHECK GUESS=READ SCRF=CHECK GENCHK RB3LYP $/ 6-311+G(D, P) \quad F$ $R E Q \backslash \backslash$ from $\backslash \backslash 0,1 \backslash C,-0.5367961031,1.3040371692,0.6696430331 \backslash C,-0.53694864$ $53,1.303507836,-0.6709319959 \backslash C,-1.1339306284,-0.0088893537,1.115630904$ $2 \backslash C,-1.1345132566,-0.0096328423,-1.1157477657 \backslash C,-0.1298951039,-1.19603$ $51185,0.7850520525 \backslash C,-2.1860352959,-0.2308735388,0.0002951906 \backslash C, 1.1111$ $869785,-1.2321858503,-0.0003580406 \backslash C,-0.1304019483,-1.1966008025,-0.78$ $49299397 \backslash \mathrm{N}, 2.1508823258,0.3996359436,-0.0006103173 \backslash \mathrm{N}, 3.1764513395,0.80$ $94815568,0.0017655323 \backslash \mathrm{H},-0.0967546462,2.0433368701,1.3261468768 \backslash \mathrm{H},-0.0$ $970879712,2.0423100276,-1.328117442 \backslash \mathrm{H},-1.4677906942,-0.0622713209,2.15$ $0546998 \backslash \mathrm{H},-1.4689267338,-0.0636742978,-2.1504518167 \backslash \mathrm{H},-0.2506552431,-2$ $.0482315241,1.4522576034 \backslash \mathrm{H},-2.6065279791,-1.2396774957,0.0007533637 \backslash \mathrm{H}$, $-2.9879172551,0.513717842,0.0002566448 \backslash \mathrm{H},-0.251671117,-2.0492975982,-1$ $.4513993649 \backslash \backslash$ Version=AM64L-G03RevC . 02 \State $=1-\mathrm{A} \backslash \mathrm{HF}=-419.0699821 \backslash \mathrm{RMSD}=4$ $.202 e-09 \backslash \mathrm{RMSF}=2.045 e-05 \backslash \mathrm{Dipole}=-1.0525638,0.4038117,-0.0000747$

Sum of electronic and zero-point Energies= Sum of electronic and thermal Energies= Sum of electronic and thermal Enthalpies= Sum of electronic and thermal Free Energies=

$-418.931141$ $-418.923243$ $-418.922299$ $-418.963977$

anti:

$1 \backslash 1 \backslash G I N C-N O D E 163 \backslash$ Freq $\backslash R B 3 L Y P \backslash 6-311+G(d, p) \backslash C 8 H 8 N 2 \backslash M I E U S S E T \backslash 24-J a n-2006 \backslash$ $0 \backslash \backslash \# N$ GEOM=ALLCHECK GUESS=READ SCRF=CHECK GENCHK RB3LYP/6-311+G (D, P) F $R E Q \backslash \backslash f r o m \backslash \backslash 0,1 \backslash C,-0.8406491493,1.7073608469,0.6686845869 \backslash C,-0.84049915$ $94,1.706141317,-0.6709737044 \backslash \mathrm{C},-1.2471925315,0.3046391675,1.1283179216$ $\backslash C,-1.2469064941,0.3025963122,-1.12836041 \backslash \mathrm{C},-0.0670731057,-0.649361593$ $8,0.7479105795 \backslash \mathrm{C},-2.2492520169,-0.0551144934,0.0001460725 \backslash \mathrm{C}, 1.06568528$ $46,0.0682972443,-0.0001807195 \backslash C,-0.0671039033,-0.6511680575,-0.7460620$ $231 \backslash \mathrm{N}, 2.488332074,-1.2360133613,0.0013671689 \backslash \mathrm{N}, 3.5842243011,-1.3710434$ $431,-0.0009785597 \backslash \mathrm{H},-0.492677705,2.4987441821,1.3190633058 \backslash \mathrm{H},-0.492396$ $812,2.4963382567,-1.3227026071 \backslash \mathrm{H},-1.5829786398,0.2088589196,2.15919148$ $95 \backslash \mathrm{H},-1.5824393139,0.2053220937,-2.1591793613 \backslash \mathrm{H}, 0.1381186875,-1.519745$ 
$3916,1.3567435087 \backslash \mathrm{H},-2.5425927623,-1.107866891,0.0010542084 \backslash \mathrm{H},-3.13232$ $90718,0.590355702,-0.0006384407 \backslash \mathrm{H}, 0.1373474451,-1.522953699,-1.3531461$ $892 \backslash \backslash$ Version=AM64L-G03RevC.02\State $=1-\mathrm{A} \backslash \mathrm{HF}=-419.0722012 \backslash \mathrm{RMSD}=3.966 \mathrm{e}-09$

$\backslash \mathrm{RMSF}=2.101 \mathrm{e}-05 \backslash \mathrm{Dipole}=-0.6592637,-0.3997278,0.0006501$

Sum of electronic and zero-point Energies=

Sum of electronic and thermal Energies=

Sum of electronic and thermal Enthalpies=

Sum of electronic and thermal Free Energies=

\author{
$-418.933476$ \\ $-418.925504$ \\ $-418.924560$ \\ $-418.966425$
}

\title{
$($ endo-6 $\rightarrow$ 27), NImag $=1$
}

$1 \backslash 1 \backslash$ GINC-NODE122 Freq \UB3LYP \6-311+G (d,p) \C8H8N2 \MIEUSSET \10-May-2006

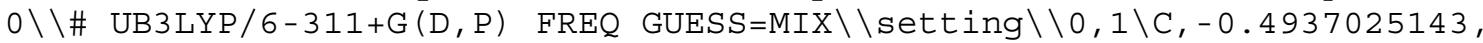
$1.1252779714,-1.4475611714 \backslash \mathrm{C},-1.5775295143,0.6488159714,-0.8342171714 \backslash$ $C,-1.3542805143,0.6817789714,0.6760008286 \backslash C,-0.5350365143,-0.516340028$ $6,1.1209788286 \backslash \mathrm{C}, 1.3104834857,0.2937639714,0.0753988286 \backslash \mathrm{C}, 0.5450024857$ $, 1.5163279714,-0.3986011714 \backslash \mathrm{C},-0.4043215143,1.9220729714,0.7780008286 \backslash$ $\mathrm{C}, 0.5450124857,-0.9370070286,0.2343298286 \backslash \mathrm{N}, 0.8869304857,-2.1383970286$ $,-0.0926081714 \backslash \mathrm{N}, 1.2060354857,-3.2075670286,-0.3557751714 \backslash \mathrm{H},-0.3132965$ $143,1.1501189714,-2.5147741714 \backslash \mathrm{H},-2.4480825143,0.2118369714,-1.3067621$ $714 \backslash \mathrm{H},-2.2584545143,0.7812839714,1.2811408286 \backslash \mathrm{H},-0.4826285143,-0.73700$ $20286,2.1869278286 \backslash \mathrm{H}, 2.2269954857,0.4467879714,0.6446588286 \backslash \mathrm{H}, 1.230635$ $4857,2.3141479714,-0.6934411714 \backslash \mathrm{H},-0.9310875143,2.8618269714,0.5769388$ $286 \backslash \mathrm{H}, 0.1113894857,1.9846029714,1.7380168286 \backslash \backslash$ Version=AM64L-G03RevC . 02 $\backslash$ State $=1-A \backslash H F=-419.0658064 \backslash S 2=0.560187 \backslash S 2-1=0 . \backslash S 2 A=0.014893 \backslash R M S D=8.803$

$e-09 \backslash \mathrm{RMSF}=4.839 e-05 \backslash \mathrm{Dipole}=-0.2769032,1.1253357,0.3736844 \backslash$

Sum of electronic and zero-point Energies=

Sum of electronic and thermal Energies=

Sum of electronic and thermal Enthalpies=

Sum of electronic and thermal Free Energies=

$$
\begin{aligned}
& -418.927279 \\
& -418.919383 \\
& -418.918439 \\
& -418.959972
\end{aligned}
$$

\section{exo-3-Diazotricyclo[3.2.1.0 $\left.{ }^{2,4}\right]$ oct-6-ene (exo-6)}

anti $(\mathrm{NImag}=0)$ :

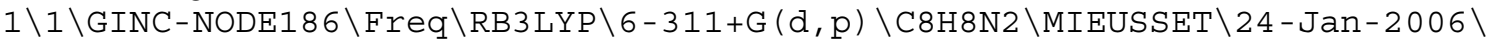

$0 \backslash \backslash \# \mathrm{~N}$ GEOM=ALLCHECK GUESS=READ SCRF=CHECK TEST GENCHK RB3LYP/6-311+G(D

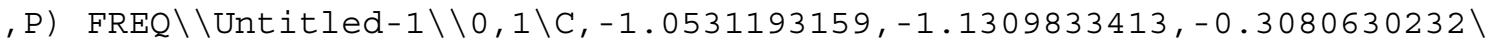
C, $-1.0531354097,1.1310524127,-0.3077193906 \backslash \mathrm{C}, 1.3080424816,0.0000602204$ $,-0.2717418347 \backslash \mathrm{C}, 0.2396196486,0.757662047,0.4730535279 \backslash \mathrm{C}, 0.2396556304$, $-0.7578537592,0.4727886749 \backslash \mathrm{C},-1.1115439627,0.0001987901,-1.3644259353 \backslash$ $\mathrm{H},-1.1024479242,2.1580095581,-0.6669746798 \backslash \mathrm{H}, 0.4972365954,1.3104031185$ $, 1.3708443597 \backslash \mathrm{H}, 0.497298723,-1.3108939217,1.3703877096 \backslash \mathrm{H},-2.0552456878$ $, 0.0002786038,-1.9095111159 \backslash \mathrm{H},-1.1024368665,-2.157828801,-0.6676357218$ $\backslash \mathrm{H},-0.270422859,0.000313342,-2.0576565837 \backslash \mathrm{N}, 2.5745380276,0.0000417103$, $-0.006681003 \backslash \mathrm{N}, 3.7093324034,0.0000411605,0.0891324954 \backslash \mathrm{C},-2.1880057819$, $0.6691361017,0.6160740207 \backslash \mathrm{H},-2.8063005943,1.3261794169,1.214132034 \backslash \mathrm{C},-$ $2.1879428301,-0.669350466,0.6159363378 \backslash \mathrm{H},-2.8061971644,-1.3265734444,1$ $.2138392869 \backslash \backslash$ Version=AM64L-G03RevC.02 \State $=1-A \backslash H F=-419.1149704 \backslash$ RMSD $=4$

$.035 e-09 \backslash \mathrm{RMSF}=4.195 e-05 \backslash \mathrm{Dipole}=-0.8708056,-0.0000734,0.3493731 \backslash$

Sum of electronic and zero-point Energies=

Sum of electronic and thermal Energies=

Sum of electronic and thermal Enthalpies=

Sum of electronic and thermal Free Energies=

$-418.971655$

$-418.964381$

$-418.963436$

$-419.003573$

TS $($ NImag $=1):$

$1 \backslash 1 \backslash$ GINC-NODE0 57 $\backslash$ Freq $\backslash R B 3 L Y P \backslash 6-311+G(d, p) \backslash C 8 H 8 N 2 \backslash M I E U S S E T \backslash 24-J a n-2006 \backslash$ $0 \backslash \backslash \# \mathrm{~N}$ GEOM=ALLCHECK GUESS=READ SCRF=CHECK TEST GENCHK RB3LYP/6-311+G(D

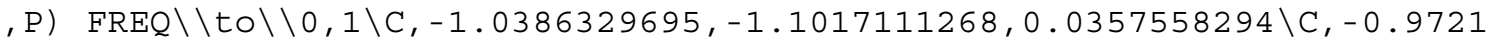
$702866,1.1608791771,0.034838347 \backslash C, 1.3479481632,-0.0396437229,-0.124855$ $1112 \backslash \mathrm{C}, 0.3989866257,0.7590698883,0.6642243303 \backslash \mathrm{C}, 0.3537175313,-0.780559$ $5892,0.6648777639 \backslash \mathrm{C},-1.180496388,0.0343153506,-1.0072318366 \backslash \mathrm{H},-1.03674$ 
$99594,2.1894464398,-0.3175564949 \backslash \mathrm{H}, 0.7447600584,1.2998222282,1.5413357$ $886 \backslash \mathrm{H}, 0.6671377823,-1.3398691212,1.5425098076 \backslash \mathrm{H},-2.1787687225,0.063482$ $7846,-1.4431586255 \backslash \mathrm{H},-1.1634959085,-2.1250030112,-0.3157949129 \backslash \mathrm{H},-0.43$ $03469529,0.0119767002,-1.7985509115 \backslash N, 2.3548450068,-0.0695274124,-0.88$ $9633857 \backslash \mathrm{N}, 3.2727463617,-0.0970396418,-1.5792599892 \backslash \mathrm{C},-2.0059012271,0.7$ $288827106,1.0811260808 \backslash \mathrm{H},-2.5340932668,1.4014794229,1.7445739742 \backslash \mathrm{C},-2$. $0450908098,-0.6089795015,1.0817762277 \backslash \mathrm{H},-2.6117464441,-1.2488851814,1$. $7458285098 \backslash \backslash$ Version=AM64L-G03RevC.02\State $=1-\mathrm{A} \backslash \mathrm{HF}=-419.111231 \backslash \mathrm{RMSD}=3.8$ $08 e-09 \backslash \mathrm{RMSF}=1.069 e-05 \backslash \mathrm{Dipole}=-0.9418791,0.0280154,0.6399632$

Sum of electronic and zero-point Energies=

$-418.968039$

Sum of electronic and thermal Energies=

Sum of electronic and thermal Enthalpies=

Sum of electronic and thermal Free Energies=

$-418.961473$

$-418.960529$

$-418.999114$

syn $(\mathrm{NImag}=0):$

$1 \backslash 1 \backslash$ GINC-NODE091 \Freq \RB3LYP \6-311+G(d,p) \C8H8N2 \MIEUSSET $24-J a n-2006 \backslash$ $0 \backslash \backslash \# \mathrm{~N}$ GEOM=ALLCHECK GUESS=READ SCRF=CHECK TEST GENCHK RB3LYP/6-311+G (D

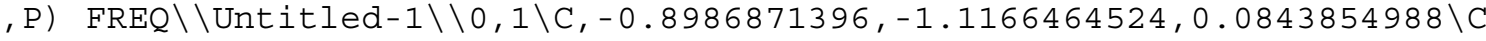
$,-0.8456656049,1.1433247436,0.0531534582 \backslash \mathrm{C}, 1.5281893536,-0.0434609328$, $0.1500379153 \backslash C, 0.4620134538,0.7568551754,0.8098616263 \backslash \mathrm{C}, 0.427009914,-0$ $.771460984,0.8310069224 \backslash \mathrm{C},-0.9704100466,0.0011657325,-0.9848940661 \backslash \mathrm{H},-$ $0.8850877275,2.1666560402,-0.3171872498 \backslash \mathrm{H}, 0.7221845947,1.319155013,1.7$ $020303036 \backslash \mathrm{H}, 0.6584421293,-1.3190367224,1.7400830835 \backslash \mathrm{H},-1.9395948796,0$. $0170755779,-1.4830772815 \backslash \mathrm{H},-0.9863847602,-2.1469778668,-0.2572128764 \backslash \mathrm{H}$ $,-0.1852795699,-0.0277536672,-1.7392072143 \backslash \mathrm{N}, 2.1069343231,-0.061720724$ $3,-0.9910585475 \backslash N, 2.6495260041,-0.0252852537,-1.9951499499 \backslash \mathrm{C},-1.962162$ $0731,0.7217426148,1.0172053242 \backslash \mathrm{H},-2.5478119274,1.4017728175,1.62232549$ $34 \backslash \mathrm{C},-1.9937903101,-0.616617773,1.0354329654 \backslash \mathrm{H},-2.6106754323,-1.251262$ $0915,1.6585673557 \backslash \backslash V e r s i o n=A M 64 L-G 03$ RevC .02 \State $=1-A \backslash H F=-419.1129388 \backslash$

$\mathrm{RMSD}=2.565 e-09 \backslash \mathrm{RMSF}=1.560 e-03 \backslash \mathrm{Dipole}=-0.8636991,0.0068406,0.5849407 \backslash$

Sum of electronic and zero-point Energies=

Sum of electronic and thermal Energies=

Sum of electronic and thermal Enthalpies=

Sum of electronic and thermal Free Energies=

$-418.969213$

$-418.961995$

$-418.961051$

$-419.001141$

\section{$($ exo-6 $\rightarrow$ exo-7), NImag $=1$}

anti :

$1 \backslash 1 \backslash$ GINC-NODE106\Freq \RB3LYP \6-311+G(d,p) \C8H8N2 \MIEUSSET $24-J a n-2006 \backslash$ $0 \backslash \backslash \# \mathrm{~N}$ GEOM=ALLCHECK GUESS=READ SCRF=CHECK TEST GENCHK RB3LYP/6-311+G (D

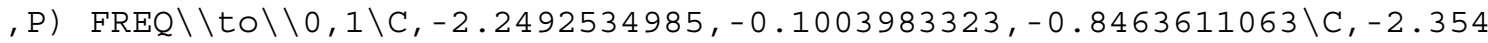
$9020619,0.1264095389,0.473132491 \backslash \mathrm{C},-0.9032014658,0.4476949327,-1.29785$ $61789 \backslash \mathrm{C},-1.0807576227,0.8288785815,0.9194655986 \backslash \mathrm{C}, 0.1610542604,-0.5175$ $106594,-0.6554451089 \backslash \mathrm{C},-0.7877255881,1.664833666,-0.3493163345 \backslash \mathrm{C}, 1.292$ $5960315,0.2061001174,0.0680736367 \backslash \mathrm{C}, 0.0439943954,-0.2661826284,0.80655$ $17833 \backslash \mathrm{N}, 2.612057355,-1.1549966122,0.4077100671 \backslash \mathrm{N}, 3.6950076697,-1.33628$ $71684,0.5258324779 \backslash \mathrm{H},-2.9305228461,-0.6677098379,-1.4679380152 \backslash \mathrm{H},-3.13$ $88975905,-0.2203682595,1.1345129061 \backslash \mathrm{H},-0.7669054298,0.6234701069,-2.36$ $36033665 \backslash \mathrm{H},-1.1062284932,1.3519559144,1.8739450622 \backslash \mathrm{H}, 0.311190128,-1.49$ $29401244,-1.0995565075 \backslash \mathrm{H}, 0.2071900372,2.1232421395,-0.3484553086 \backslash \mathrm{H},-1$. $5537601515,2.4169525663,-0.5399509524 \backslash \mathrm{H}, 0.0976524712,-1.034567339,1.56$ $67796813 \backslash \backslash$ Version=AM64L-G03RevC.02\State $=1-\mathrm{A} \backslash \mathrm{HF}=-419.0772027 \backslash \mathrm{RMSD}=5.11$ $0 e-09 \backslash \mathrm{RMSF}=3.252 e-06 \backslash \mathrm{Dipole}=-0.6719879,-0.4010846,0.0151344$

Sum of electronic and zero-point Energies=

$-418.938206$ Sum of electronic and thermal Energies= Sum of electronic and thermal Enthalpies= Sum of electronic and thermal Free Energies= $-418.930320$ $-418.929376$ $-418.971070$

syn:

$1 \backslash 1 \backslash$ GINC-NODE113 $\backslash$ Freq $\backslash R B 3 L Y P \backslash 6-311+G(d, p) \backslash C 8 H 8 N 2 \backslash M I E U S S E T \backslash 25-J a n-2006 \backslash$ $0 \backslash \backslash \# \mathrm{~N}$ GEOM=ALLCHECK GUESS=READ SCRF=CHECK TEST GENCHK RB3LYP/6-311+G(D 
,P) FREQ $\backslash \backslash$ to $\backslash \backslash 0,1 \backslash \mathrm{C}, 1.8590571814,-0.6685224056,-1.3408221377 \backslash \mathrm{C}, 1.85907$ $10368,0.6683024375,-1.3409021516 \backslash \mathrm{C}, 0.884894993,-1.1167709387,-0.241890$ $9271 \backslash \mathrm{C}, 0.8848262805,1.1166215619,-0.2421090837 \backslash \mathrm{C},-0.540208721,-0.78312$ $67243,-0.790761211 \backslash \mathrm{C}, 1.1298928501,0.0000136673,0.7874685594 \backslash \mathrm{C},-1.63552$ $40771,0.0000834437,-0.1887595441 \backslash \mathrm{C},-0.5401745452,0.7827744263,-0.79118$ $10329 \backslash \mathrm{N},-1.8414687068,0.0008535744,1.67484876 \backslash \mathrm{N},-2.5851196314,-0.00016$ $58404,2.494141896 \backslash \mathrm{H}, 2.3718431533,-1.3328636063,-2.0238869943 \backslash \mathrm{H}, 2.37186$ $27681,1.3325520444,-2.0240564605 \backslash \mathrm{H}, 0.9789436629,-2.1490960593,0.093905$ $8843 \backslash \mathrm{H}, 0.9786315019,2.148995392,0.0936031297 \backslash \mathrm{H},-0.8674024118,-1.428815$ $8902,-1.6053263667 \backslash \mathrm{H}, 0.4493903278,0.0000655313,1.6351235147 \backslash \mathrm{H}, 2.158812$ $2738,0.0000934117,1.1477234238 \backslash \mathrm{H},-0.8669728997,1.4280022299,-1.6062755$ $509 \backslash \backslash$ Version=AM64L-G03RevC .02\State $=1-A \backslash H F=-419.0727561 \backslash$ RMSD=2 $026 e-09$

$\backslash$ RMSF $=1.160 e-05 \backslash$ Dipole $=0.9517887,0.0000211,-0.1360709 \backslash$

Sum of electronic and zero-point Energies=

Sum of electronic and thermal Energies=

Sum of electronic and thermal Enthalpies=

Sum of electronic and thermal Free Energies=

$-418.933517$

$-418.925677$

$-418.924733$

$-418.966386$

\section{$($ exo-6 $\rightarrow$ 26), NImag $=1$}

$1 \backslash 1 \backslash$ GINC-NODE104 $\backslash$ Freq $\backslash R B 3 L Y P \backslash 6-311+G(d, p) \backslash C 8 H 8 N 2 \backslash M I E U S S E T \backslash 24-J a n-2006 \backslash$ $0 \backslash \backslash \# \mathrm{~N}$ GEOM=ALLCHECK GUESS=READ SCRF=CHECK GENCHK RB3LYP/6-311+G (D,P) F $R E Q \backslash \backslash f r o m \backslash \backslash 0,1 \backslash C,-0.7689971592,-1.0845568021,0.5300519442 \backslash C,-1.5539275$ $494,0.4210013302,-1.0108276727 \backslash C, 1.5011838327,-0.1080652558,-0.4595747$ $749 \backslash \mathrm{C},-0.4916309543,1.114898635,-0.2596981022 \backslash \mathrm{C}, 0.395444954,0.12073862$ $64,0.4486376165 \backslash \mathrm{C},-1.2374500247,-1.0746758813,-0.9412318046 \backslash \mathrm{H},-1.99950$ $17133,0.8731588561,-1.8885184428 \backslash \mathrm{H},-0.2355270342,2.1557333873,-0.40793$ $84814 \backslash \mathrm{H}, 0.6606905991,0.4217656441,1.4674537997 \backslash \mathrm{H},-2.1241770916,-1.6932$ $579639,-1.0971922794 \backslash \mathrm{H},-0.4110392269,-2.013534794,0.9606509402 \backslash \mathrm{H},-0.44$ $56549901,-1.3617375653,-1.6333220364 \backslash N, 2.7152724928,0.0546712168,-0.07$ $3580418 \backslash \mathrm{N}, 3.8433224223,0.1562703113,0.1146770944 \backslash \mathrm{C},-2.1206592676,0.751$ $142865,0.3783128455 \backslash \mathrm{H},-2.7599636726,1.594212546,0.5922941865 \backslash \mathrm{C},-1.8105$ $947852,-0.3172626195,1.222607662 \backslash \mathrm{H},-2.0752055543,-0.3922561927,2.26922$ 92958 \Version=AM64L-G03RevC.02\State $=1-A \backslash H F=-419.0544856 \backslash$ RMSD $=2.708 e-$

$09 \backslash \mathrm{RMSF}=2.388 e-05 \backslash \mathrm{Dipole}=-2.2398536,0.3111706,0.4918988$

Sum of electronic and zero-point Energies= Sum of electronic and thermal Energies= Sum of electronic and thermal Enthalpies= sum of electronic and thermal Free Energies=

$-418.914214$

$-418.906703$

$-418.905759$

$-418.946557$

\section{$($ exo-6 $\rightarrow$ 27), NImag $=1$}

$1 \backslash 1 \backslash$ GINC-NODE154 \Freq \UB3LYP \6-311+G (d,p) \C8H8N2 \MIEUSSET $09-M a y-2006 \backslash$

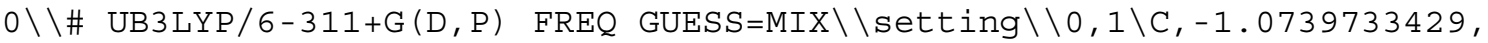
$1.1661550429,-0.0371025714 \backslash \mathrm{C}, 0.3891026571,1.0842420429,-0.4317965714 \backslash \mathrm{C}$ $, 0.3822926571,-1.1542099571,-0.1875395714 \backslash \mathrm{C},-1.0809993429,-1.140261957$ $1,0.2145134286 \backslash \mathrm{C}, 1.1777076571,0.0146610429,0.1677764286 \backslash \mathrm{N}, 2.4098686571$ $, 0.0530580429,0.5538744286 \backslash \mathrm{N}, 3.5141466571,0.0840930429,0.8665644286 \backslash \mathrm{H}$, $-1.3682993429,2.1891120429,0.2179264286 \backslash \mathrm{H}, 0.7126966571,1.5442850429,-1$ $.3642225714 \backslash \mathrm{H}, 0.7027346571,-1.8069209571,-0.9981215714 \backslash \mathrm{H},-1.3813183429$ $,-2.0823799571,0.6839094286 \backslash \mathrm{C},-1.3035313429,0.1214570429,1.0770924286 \backslash$ $\mathrm{H},-0.6038103429,0.2101230429,1.9094684286 \backslash \mathrm{H},-2.3269673429,0.1654350429$ $, 1.4517154286 \backslash \mathrm{C},-1.9428983429,0.5483090429,-1.1592615714 \backslash \mathrm{H},-2.39972034$ $29,1.1270300429,-1.9515555714 \backslash C,-1.9469683429,-0.7736689571,-1.0150175$ $714 \backslash \mathrm{H},-2.4078163429,-1.5068419571,-1.6641785714 \backslash \backslash$ Version=AM64L-G03RevC $.02 \backslash$ State $=1-A \backslash H F=-419.0639567 \backslash S 2=0.596669 \backslash S 2-1=0 . \backslash S 2 A=0.017389 \backslash R M S D=5$.

$470 e-09 \backslash \operatorname{RMSF}=7.860 e-06 \backslash \mathrm{Dipole}=-1.1206892,-0.043993,-0.4312811 \backslash$

Sum of electronic and zero-point Energies= Sum of electronic and thermal Energies= Sum of electronic and thermal Enthalpies= Sum of electronic and thermal Free Energies=

$-418.925520$

$-418.917616$

$-418.916672$ $-418.958148$ 
endo-Tricyclo[3.2.1.0 $\left.{ }^{2,4}\right]$ oct-6-en-3-ylidene (endo-7), NImag = 0

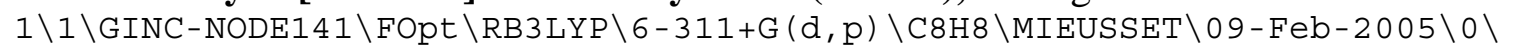
$\backslash \#$ RB3LYP/6-311+G(D,P) OPT FREQ TEST \\setting \\0,1\C,-0.6701419901,1.3 $857776255,0.1921041343 \backslash \mathrm{C},-0.3531147947,0.5355997445,-1.0596245404 \backslash \mathrm{C},-1$ $.1098728292,-0.7542554937,-0.6963375849 \backslash C,-1.0330962745,-0.901828104,0$ $.6364109583 \backslash \mathrm{C},-0.2238050815,0.2871240756,1.1839899244 \backslash \mathrm{C}, 1.1400697136,0$ $.1743949198,-0.7988901129 \backslash C, 1.2254978053,0.0103479552,0.6829846242 \backslash C, 1$ $.2766057766,-1.2230437134,-0.2089686504 \backslash \mathrm{H},-1.7389521178,1.6104021439,0$ $.2786023253 \backslash \mathrm{H},-0.0759065378,2.299639973,0.2590669145 \backslash \mathrm{H},-0.5582793845,0$ $.9645105793,-2.0379242158 \backslash \mathrm{H},-1.4998408806,-1.4689188626,-1.4084330897 \backslash$ $\mathrm{H},-1.3486895432,-1.7594278888,1.2150438425 \backslash \mathrm{H},-0.3112326927,0.489708340$ $5,2.2490921706 \backslash \mathrm{H}, 1.9310263719,0.5413739514,-1.4432394601 \backslash \mathrm{H}, 2.089020831$ $2,0.2380097062,1.2977789967 \backslash \backslash$ Version $=x 86-$ Linux-G03RevB. $02 \backslash$ State $=1-A \backslash H F$ $=-309.520292 \backslash \mathrm{RMSD}=4.429 \mathrm{e}-09 \backslash \mathrm{RMSF}=2.340 \mathrm{e}-05 \backslash \mathrm{Dipole}=-0.4016119,0.8986623$ , $0.1226198 \backslash \mathrm{PG}=\mathrm{C} 01[\mathrm{X}(\mathrm{C} 8 \mathrm{H} 8)] \backslash \backslash \backslash$

Sum of electronic and zero-point Energies= Sum of electronic and thermal Energies= Sum of electronic and thermal Enthalpies= Sum of electronic and thermal Free Energies=

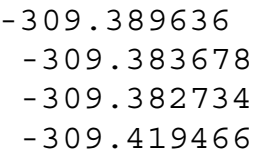

\section{$($ endo $-7 \rightarrow$ 18), NImag $=1$}

$1 \backslash 1 \backslash G I N C-N O D E 081 \backslash F T S \backslash R B 3 L Y P \backslash 6-311+G(d, p) \backslash C 8 H 8 \backslash M I E U S S E T \backslash 25-O c t-2005 \backslash 0 \backslash \backslash$ \# RB3LYP/6-311+G(D,P) OPT (QST2) FREQ \\from \\0,1\H,-0.1759883607,-0.797 $8138044,2.1520900344 \backslash C,-0.1780544804,-0.4606719473,1.1173227252 \backslash C, 1.13$ $37749894,0.3548995909,-0.7692866518 \backslash C,-0.1780496349,-0.4606548796,-1.1$ $173242355 \backslash \mathrm{C}, 1.1337520279,0.3549024689,0.7692952574 \backslash \mathrm{H}, 1.963051291,0.234$ $6930055,-1.4627021525 \backslash \mathrm{H},-0.1759823175,-0.7977740061,-2.1520986211 \backslash \mathrm{H}, 1$. $9630174175,0.2347546833,1.4627340171 \backslash \mathrm{C},-0.1476325724,-1.5282303457,-0$. $0000058186 \backslash \mathrm{H},-1.0371654453,-2.165892574,-0.0000131577 \backslash \mathrm{H}, 0.7607539036,-$ $2.1342961641,-0.000009981 \backslash C, 1.1253759177,1.6159913748,-0.0000072423 \backslash C$, $-1.3816595271,0.3599615115,0.6685377142 \backslash \mathrm{H},-2.0263942637,0.9246664527,1$ $.3289620003 \backslash \mathrm{C},-1.3816563918,0.3599671764,-0.6685330275 \backslash \mathrm{H},-2.0263941958$ $, 0.924672708,-1.3289544656 \backslash \backslash$ Version=AM64L-G03RevC.02 $\backslash$ State $=1-\mathrm{A} \backslash \mathrm{HF}=-309$ $.519915 \backslash \mathrm{RMSD}=2.117 \mathrm{e}-09 \backslash \mathrm{RMSF}=6.326 \mathrm{e}-05 \backslash \mathrm{Dipole}=-0.2550446,-1.1350188,0.0$ $000047 \backslash \mathrm{PG}=\mathrm{C} 01 \quad[\mathrm{X}(\mathrm{C} 8 \mathrm{H} 8)] \backslash \backslash @$

Sum of electronic and zero-point Energies= Sum of electronic and thermal Energies= Sum of electronic and thermal Enthalpies= Sum of electronic and thermal Free Energies=

-309.389773
-309.384420
-309.383476
-309.418721

\section{exo-Tricyclo[3.2.1.0 $\left.{ }^{2,4}\right]$ oct-6-en-3-ylidene (exo-7), NImag $=0$}

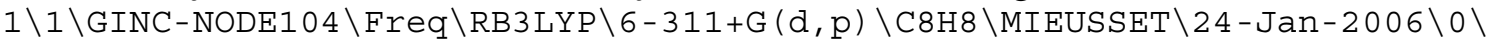
$\backslash \# N$ GEOM=ALLCHECK GUESS=READ SCRF=CHECK TEST GENCHK RB3LYP/6-311+G (D, P

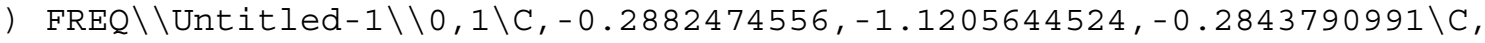
$-0.2882899934,1.1206381283,-0.2840307485 \backslash C, 2.1286752649,0.0000382012$, $0.1932393903 \backslash C, 1.0488989146,0.7608278275,0.4752908803 \backslash C, 1.0488685868,-$ $0.7608728946,0.4751141346 \backslash \mathrm{C},-0.3173804281,0.0002044513,-1.3435899983 \backslash \mathrm{H}$ $,-0.30106972,2.1531688636,-0.6289126478 \backslash \mathrm{H}, 1.3518833089,1.4477599239,1$. $2619380259 \backslash \mathrm{H}, 1.3518578102,-1.4479771822,1.2616086642 \backslash \mathrm{H},-1.2438612912,0$ $.0002916819,-1.9182236118 \backslash \mathrm{H},-0.3009617448,-2.1529908098,-0.6295785226 \backslash$ $\mathrm{H}, 0.5534726049,0.0003201959,-2.0078138165 \backslash \mathrm{C},-1.4395139149,0.6707127659$ $, 0.6010813086 \backslash \mathrm{H},-2.0660983657,1.328208779,1.1895581882 \backslash \mathrm{C},-1.4395305062$ $,-0.6709944537,0.6008155031 \backslash \mathrm{H},-2.0661054102,-1.3287188932,1.1890481786$ $\backslash \backslash$ Version=AM64L-G03RevC.02\State $=1-A \backslash H F=-309.5287396 \backslash$ RMSD $=8.366 \mathrm{e}-10 \backslash \mathrm{RM}$

$\mathrm{SF}=3.077 \mathrm{e}-05 \backslash \mathrm{Dipole}=-1.1861132,-0.000039,0.3481295 \backslash$

Sum of electronic and zero-point Energies= $\quad-309.397950$

Sum of electronic and thermal Energies= $\quad-309.392044$ 
Sum of electronic and thermal Enthalpies=

Sum of electronic and thermal Free Energies=
$-309.391100$

$-309.427409$

\section{$($ exo-7 $\rightarrow$ 18), NImag $=1$}

$1 \backslash 1 \backslash G I N C-N O D E 166 \backslash F r e q \backslash R B 3 L Y P \backslash 6-311+G(d, p) \backslash C 8 H 8 \backslash M I E U S S E T \backslash 25-J a n-2006 \backslash 0 \backslash$ $\backslash \# N$ GEOM=ALLCHECK GUESS=READ SCRF=CHECK TEST GENCHK RB3LYP/6-311+G(D,P ) $\mathrm{FREQ} \backslash \backslash \mathrm{tO} \backslash \backslash 0,1 \backslash \mathrm{C},-0.4892554835,-0.3772388539,1.0144577475 \backslash \mathrm{C}, 1.2175852$ $256,0.1577359337,-0.6645558082 \backslash C,-0.1813710452,0.8322279065,-0.8277580$ $267 \backslash C, 0.9949110675,-0.7166478644,0.6673095896 \backslash C,-1.2048371096,-0.25201$ $42645,-1.1738276771 \backslash C, 2.1078883743,0.199735595,0.4834352345 \backslash C,-0.56995$ $29754,1.1112205422,0.6342942245 \backslash C,-1.3897449818,-0.9783474328,-0.06752$ $63315 \backslash \mathrm{H},-0.7188557223,-0.6570509687,2.0423667289 \backslash \mathrm{H}, 1.7063287398,-0.101$ $2331211,-1.6043388985 \backslash \mathrm{H},-0.1246665196,1.6773535129,-1.5134380942 \backslash \mathrm{H}, 1.2$ $884123989,-1.7420598323,0.8943289308 \backslash \mathrm{H},-1.6244333348,-0.4180952516,-2$. $1570605393 \backslash \mathrm{H}, 0.1391366888,1.7466557388,1.1699782475 \backslash \mathrm{H},-1.5836352419,1$. $5024301166,0.7216847199 \backslash \mathrm{H},-1.9936254401,-1.868029566,0.0515051893 \backslash \backslash \mathrm{Ver}$ sion $=\mathrm{AM} 64 \mathrm{~L}-\mathrm{G} 03 \mathrm{RevC} .02 \backslash$ State $=1-\mathrm{A} \backslash \mathrm{HF}=-309.5284807 \backslash \mathrm{RMSD}=8.891 \mathrm{e}-10 \backslash \mathrm{RMSF}=3$.

$152 e-05 \backslash$ Dipole $=-1.2735396,-0.2240799,-0.3600035 \backslash$

Sum of electronic and zero-point Energies= Sum of electronic and thermal Energies= Sum of electronic and thermal Enthalpies= $-309.397851$ $-309.392508$ $-309.391564$ Sum of electronic and thermal Free Energies= $\quad-309.426821$

\section{Bicyclo[4.1.0]hept-3-en-7-ylidene (8), NImag $=0$}

$1 \backslash 1 \backslash$ GINC-NODE156 $\backslash$ Freq $\backslash R B 3 L Y P \backslash 6-311+G(d, p) \backslash C 7 H 8 \backslash M I E U S S E T \backslash 03-M a r-2006 \backslash 0 \backslash$ $\backslash \# N$ GEOM=ALLCHECK GUESS=READ SCRF=CHECK GENCHK RB3LYP/6-311+G (D,P) FRE

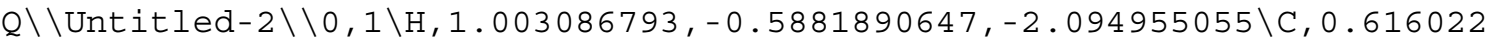
$9311,-0.475060619,-1.0836832505 \backslash C, 0.2820806922,1.2815447447,0.84595688$ $28 \backslash C,-1.4707210651,-0.4618474001,0.3473986057 \backslash C,-1.0049446748,0.542891$ $8547,1.0919541649 \backslash \mathrm{C},-0.7896932735,-1.030408798,-0.8673014124 \backslash \mathrm{C}, 1.13768$ $77853,0.6502372016,-0.249788542 \backslash \mathrm{H}, 0.8608379095,1.3419349426,1.77601349$ $79 \backslash \mathrm{H},-1.5940566272,0.8864924348,1.9378476723 \backslash \mathrm{H},-1.3989826703,-0.807961$ $9001,-1.7522227561 \backslash \mathrm{H}, 1.883773468,1.3115647484,-0.687154717 \backslash \mathrm{H}, 0.0489904$ $261,2.3155012902,0.5623995413 \backslash \mathrm{H},-2.4263466749,-0.9088636627,0.60741058$ $95 \backslash \mathrm{H},-0.7459302565,-2.1240695254,-0.792446809 \backslash \mathrm{C}, 1.6243388769,-0.745091$ $8611,-0.0106851091 \backslash \backslash$ Version=AM64L-G03RevC.02 \State $=1-A^{\prime} \backslash \mathrm{HF}=-271.445463$ $4 \backslash \mathrm{RMSD}=1.441 \mathrm{e}-09 \backslash \mathrm{RMSF}=2.606 \mathrm{e}-06 \backslash \mathrm{Dipole}=-0.5845162,0.633596,-0.4893456 \backslash$

Sum of electronic and zero-point Energies= Sum of electronic and thermal Energies= Sum of electronic and thermal Enthalpies= Sum of electronic and thermal Free Energies=
$-271.321163$

$-271.315035$

$-271.314090$

$-271.350793$

\section{Tetracyclo[3.3.0.0 $\left.{ }^{2,8} \cdot \mathbf{0}^{3,6}\right]$ octan-4-ylidene (14a), NImag $=0$}

$1 \backslash 1 \backslash G I N C-N O D E 148 \backslash F O p t \backslash R B 3 L Y P \backslash 6-311+G(d, p) \backslash C 8 H 8 \backslash M I E U S S E T \backslash 13-J a n-2005 \backslash 0 \backslash$

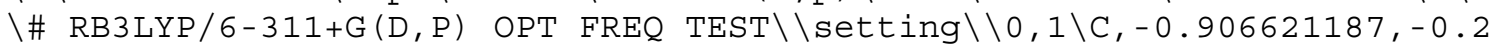
$797771857,-1.3923885008 \backslash C,-0.5477495949,-1.2823372859,-0.3100289381 \backslash C$, $-0.506969925,-0.5681785686,1.0175146741 \backslash C,-0.5152376611,0.9392642063,0$ $.7642061296 \backslash \mathrm{C},-0.1940513181,0.924752807,-0.7674397301 \backslash \mathrm{C}, 0.8193191344,-$ $0.9448276108,0.2288963533 \backslash C, 1.2089375915,0.4504349221,-0.2600880711 \backslash C$, $0.9658160864,1.0461984881,1.1272226465 \backslash \mathrm{H},-1.9868236172,-0.1299388079,-$ $1.4854763242 \backslash \mathrm{H},-0.497173036,-0.5519719438,-2.3702088857 \backslash \mathrm{H},-0.852365152$ $1,-2.321874022,-0.3258284326 \backslash \mathrm{H},-0.756514605,-1.0165717514,1.9659089922$ $\backslash \mathrm{H},-1.2914828268,1.5316182772,1.2419467793 \backslash \mathrm{H},-0.2289717449,1.881126210$ $5,-1.282774401 \backslash \mathrm{H}, 1.5267658712,-1.6641597647,0.6094970833 \backslash \mathrm{H}, 2.145906354$ $2,0.558593167,-0.8004321916 \backslash \backslash$ Version=x86-Linux-G03RevB.02 \State $=1-A \backslash H F$ $=-309.5326472 \backslash \mathrm{RMSD}=9.315 \mathrm{e}-09 \backslash \mathrm{RMSF}=7.699 \mathrm{e}-05 \backslash \mathrm{Dipole}=-0.6731497,-0.66369$ $33,-0.816113 \backslash \mathrm{PG}=\mathrm{C} 01[\mathrm{X}(\mathrm{C} 8 \mathrm{H} 8)] \backslash \backslash @$ 
Sum of electronic and zero-point Energies= Sum of electronic and thermal Energies= Sum of electronic and thermal Enthalpies= Sum of electronic and thermal Free Energies=
$-309.400569$

$-309.395029$

$-309.394084$

$-309.429833$

\section{$(\mathbf{1 4 a} \rightarrow$ 14), NImag $=1$}

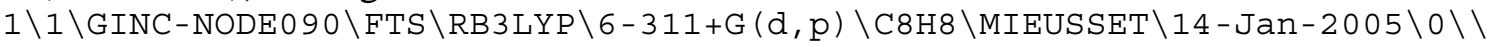

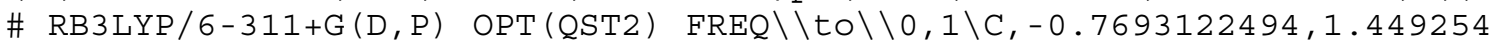
$8988,-0.3810902059 \backslash \mathrm{C},-0.5407013897,0.2691005638,-1.3049758811 \backslash \mathrm{C},-0.609$ $3682396,-1.0035152853,-0.495070956 \backslash C,-0.5702089736,-0.6433494913,0.993$ $2894122 \backslash \mathrm{C},-0.1002052763,0.8454322347,0.8590105078 \backslash \mathrm{C}, 0.7679231154,-0.38$ $96533259,-0.9388068069 \backslash \mathrm{C}, 1.2371306373,0.1629094705,0.4110565189 \backslash \mathrm{C}, 0.87$ $4674804,-1.1063519456,1.1802648436 \backslash \mathrm{H},-1.8311227316,1.6624794186,-0.222$ $9401493 \backslash \mathrm{H},-0.2692897626,2.3595305617,-0.7264507979 \backslash \mathrm{H},-0.862759906,0.24$ $43756818,-2.3392435293 \backslash \mathrm{H},-0.9689969902,-1.9493068117,-0.8689535053 \backslash \mathrm{H},-$ $1.3866998466,-0.9893651428,1.6225857488 \backslash \mathrm{H},-0.0664396815,1.4305021712,1$ $.7742514932 \backslash \mathrm{H}, 1.4234332128,-0.8819824556,-1.6399060267 \backslash \mathrm{H}, 2.2222811374$, $0.6208038587,0.4585921704 \backslash \backslash$ Version=x86-Linux-G03RevB. 02 $\backslash$ State $=1-\mathrm{A} \backslash \mathrm{HF}=-$ $309.5325941 \backslash \mathrm{RMSD}=4.862 e-09 \backslash \mathrm{RMSF}=6.787 e-05 \backslash \mathrm{Dipole}=-0.5955216,0.7988134$, $-0.7423982 \backslash \mathrm{PG}=\mathrm{CO} 1[\mathrm{X}(\mathrm{C} 8 \mathrm{H} 8)] \backslash \backslash @$

Sum of electronic and zero-point Energies= Sum of electronic and thermal Energies= Sum of electronic and thermal Enthalpies= Sum of electronic and thermal Free Energies=

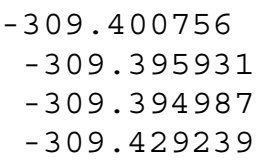

\section{Non-classical tetracyclo $\left[3.3 .0 .0^{2,8} \cdot 0^{3,6}\right]$ octan-4-ylidene (14), NImag = 0}

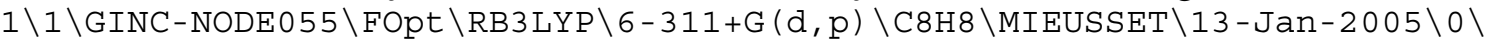

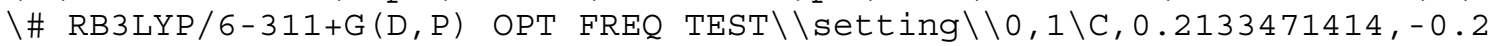
$676863304,-1.5842912873 \backslash \mathrm{C}, 0.1856632526,-1.3643934678,-0.5566285274 \backslash \mathrm{C},-$ $0.7500719349,-0.9275805316,0.5543627678 \backslash C,-1.1670733401,0.5408882,0.36$ $77732561 \backslash C,-0.0044519341,0.9582080737,-0.6847423938 \backslash C, 0.7458851777,-0$. $7973198752,0.7338147886 \backslash \mathrm{C}, 0.9393121668,0.7236080082,0.6215701623 \backslash \mathrm{C},-0$. $2526879846,1.4825215109,1.0461543862 \backslash \mathrm{H},-0.590823329,-0.3441095293,-2.3$ $225528371 \backslash \mathrm{H}, 1.1703477249,-0.1892750896,-2.1089778297 \backslash \mathrm{H}, 0.3089151598,-2$ $.4084626714,-0.8230493332 \backslash \mathrm{H},-1.3610670884,-1.6218444117,1.1147507585 \backslash \mathrm{H}$ $,-2.2102174482,0.7906785739,0.19762333 \backslash \mathrm{H},-0.0299528178,1.9220223901,-1$ $.1743865623 \backslash \mathrm{H}, 1.317506181,-1.3883538194,1.4362105591 \backslash \mathrm{H}, 1.9357563491,1$. $1498710298,0.692303 \backslash \backslash$ Version=x86-Linux-G03RevB.02 \State $=1-A \backslash H F=-309.54$ $40931 \backslash R M S D=4.488 e-09 \backslash R M S F=1.048 e-04 \backslash D i p o l e=0.1876949,-1.0743177,-0.780$ $9379 \backslash \mathrm{PG}=\mathrm{C} 01 \quad[\mathrm{X}(\mathrm{C} 8 \mathrm{H} 8)] \backslash \backslash \backslash$

Sum of electronic and zero-point Energies= Sum of electronic and thermal Energies= Sum of electronic and thermal Enthalpies= Sum of electronic and thermal Free Energies=

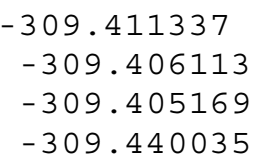

\section{$(14 \rightarrow \mathbf{1 5})$, NImag $=1$}

$1 \backslash 1 \backslash G I N C-N O D E 141 \backslash F T S \backslash R B 3 L Y P \backslash 6-311+G(d, p) \backslash C 8 H 8 \backslash M I E U S S E T \backslash 15-J a n-2005 \backslash 0 \backslash \backslash$

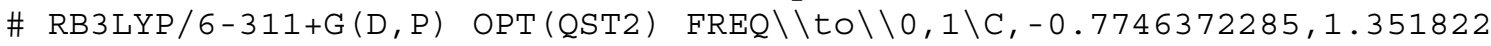
$4373,-0.3754954385 \backslash C,-0.5518152453,0.2159167876,-1.3322476103 \backslash C,-0.544$ $8375653,-1.1029052809,-0.5704195596 \backslash C,-0.5839351802,-0.9105528548,0.92$ $5003393 \backslash \mathrm{C}, 0.0739708338,0.9613062623,0.8247630327 \backslash \mathrm{C}, 0.7611243008,-0.453$ $3356183,-0.9754444909 \backslash C, 1.2761331266,0.1208350323,0.360250248 \backslash C, 0.6273$ $387651,-0.5489052856,1.5440717454 \backslash \mathrm{H},-1.8247209204,1.5192672064,-0.1222$ $424372 \backslash \mathrm{H},-0.3659450568,2.2927009038,-0.7666422184 \backslash \mathrm{H},-0.9133391336,0.25$ $51150101,-2.3539806882 \backslash \mathrm{H},-0.9490936372,-2.0004467567,-1.0218362561 \backslash \mathrm{H},-$ $1.4748939905,-1.1701887205,1.4941183031 \backslash \mathrm{H}, 0.1175311701,1.6557737976,1$. $6523138442 \backslash \mathrm{H}, 1.4251092993,-0.8696112394,-1.7207845749 \backslash \mathrm{H}, 2.2853014276,0$ $.5123009186,0.4361661081 \backslash \backslash$ Version $=x 86-$ Linux-G03RevB. 02 \State $=1-A \backslash H F=-3$ 
$09.5393583 \backslash \mathrm{RMSD}=9.091 e-09 \backslash \mathrm{RMSF}=3.695 e-05 \backslash \mathrm{Dipole}=-0.6562138,0.4539988,-$ $1.2565374 \backslash \mathrm{PG}=\mathrm{CO} 1 \mathrm{X}(\mathrm{C} 8 \mathrm{H} 8)] \backslash \backslash @$

Sum of electronic and zero-point Energies= Sum of electronic and thermal Energies= Sum of electronic and thermal Enthalpies= $-309.407766$ $-309.402838$ $-309.401894$ Sum of electronic and thermal Free Energies=

$-309.436328$

\section{$(\mathbf{1 4} \rightarrow$ endo-24), NImag $=1$}

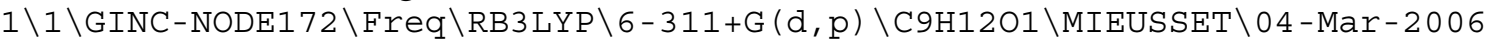
$\backslash 0 \backslash \backslash \# N$ GEOM=ALLCHECK GUESS=READ SCRF=CHECK TEST GENCHK RB3LYP/6-311+G(

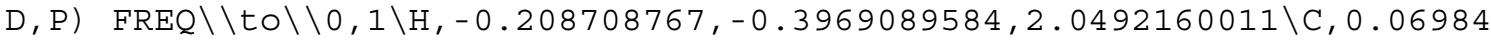
$23953,-0.258371513,1.0081377457 \backslash C, 0.5115037403,-0.6544113422,-1.009911$ $5537 \backslash \mathrm{H}, 0.6579582728,-1.1789871313,-1.9499716081 \backslash \mathrm{C},-0.6334491911,-0.964$ $1493859,-0.1059848662 \backslash 0,-2.5331056787,-0.2867770367,-0.7561898607 \backslash \mathrm{H},-1$ $.845669433,-1.2035669286,-0.399584874 \backslash C,-3.2978503643,0.1631986527,0.3$ $413664706 \backslash \mathrm{H},-3.0547900297,1.2012378473,0.6051659158 \backslash \mathrm{H},-3.1490146534,-0$ $.4631584069,1.2340417266 \backslash \mathrm{H},-4.3629079126,0.1170681663,0.082106778 \backslash \mathrm{C}, 1$. $2847811436,-1.0495573296,0.3313942198 \backslash \mathrm{H}, 1.3808234198,-2.105118836,0.56$ $0596958 \backslash \mathrm{C}, 2.5310012783,-0.1656243412,0.431886945 \backslash \mathrm{H}, 2.9364009519,-0.161$ $2634451,1.4483932678 \backslash \mathrm{C}, 1.9046361103,1.1485749684,0.0349565212 \backslash \mathrm{H}, 2.4739$ $480392,2.0689763254,-0.0206452624 \backslash \mathrm{H}, 3.3109151791,-0.4986921778,-0.2598$ $258672 \backslash \mathrm{C}, 0.7799525545,0.8604477636,-0.9419837423 \backslash \mathrm{H}, 0.4998681853,1.5236$ $225341,-1.7475396835 \backslash \mathrm{C}, 0.463347957,1.1459489734,0.5114348442 \backslash \mathrm{H},-0.0565$ $715661,2.0346686273,0.8397860277 \backslash \backslash$ Version=AM64L-G03RevC .02 \State =1 -A \H $\mathrm{F}=-425.3050595 \backslash \mathrm{RMSD}=5.666 \mathrm{e}-09 \backslash \mathrm{RMSF}=1.585 e-05 \backslash \mathrm{Dipole}=1.1811963,0.264641$ $4,0.6537748 \backslash$

Sum of electronic and zero-point Energies= Sum of electronic and thermal Energies= Sum of electronic and thermal Enthalpies= Sum of electronic and thermal Free Energies=

$$
\begin{aligned}
& -425.123093 \\
& -425.114165 \\
& -425.113221 \\
& -425.157827
\end{aligned}
$$

\section{$(14 \rightarrow$ exo-24), NImag $=1$}

$1 \backslash 1 \backslash$ GINC-NODE098\Freq \RB3LYP \6-311+G(d,p) \C9H1201 \MIEUSSET $04-$ Mar-2006 $\backslash 0 \backslash \backslash \# N$ GEOM=ALLCHECK GUESS=READ SCRF=CHECK TEST GENCHK RB3LYP/6-311+G( $\mathrm{D}, \mathrm{P}) \mathrm{FREQ} \backslash \backslash \mathrm{tO} \backslash \backslash 0,1 \backslash \mathrm{H},-0.5904668317,0.2828889953,1.8151153051 \backslash \mathrm{C},-0.0493$ $208177,-0.0216611185,0.9227892245 \backslash \mathrm{C}, 0.4200314683,-0.394894062,-1.06211$ $71853 \backslash \mathrm{H}, 0.3339119285,-0.4576710743,-2.1432969756 \backslash \mathrm{C},-0.6542735646,-0.93$ $44140255,-0.1321952883 \backslash \mathrm{O},-2.5589941342,-0.1251175998,-0.838237613 \backslash \mathrm{H},-1$ $.8791711182,-1.0438250184,-0.4885156001 \backslash \mathrm{C},-3.3955928726,0.2430015392,0$ $.2360618931 \backslash \mathrm{H},-3.1930169099,1.2672991216,0.5784655173 \backslash \mathrm{H},-3.2867598889$, $-0.4358380167,1.0957434588 \backslash \mathrm{H},-4.4426322081,0.1942374937,-0.0889873048 \backslash$ C, $2.3781389135,0.1361948227,0.3831846165 \backslash \mathrm{C}, 1.7626484479,1.4612378146,-$ $0.0104479696 \backslash \mathrm{H}, 2.2083605543,1.8856902179,-0.9153141065 \backslash \mathrm{C}, 0.3407476189$, $0.9536233744,-0.2514008316 \backslash \mathrm{H},-0.435127459,1.6403464549,-0.5721382809 \backslash \mathrm{H}$ , $3.4210266501,0.0214174971,0.6528242029 \backslash \mathrm{C}, 1.6754446059,-0.9674236606,-$ $0.3800934667 \backslash \mathrm{H}, 2.1582721555,-1.8760630208,-0.7082486069 \backslash \mathrm{C}, 1.3267407678$ $,-0.6914488369,1.0941330693 \backslash \mathrm{H}, 1.5429247727,-1.3885419275,1.8901933112 \backslash$ $\mathrm{H}, 1.8072440243,2.2057049901,0.7905756117 \backslash \backslash$ Version=AM64L-G03RevC.02\Sta $t e=1-A \backslash H F=-425.3005316 \backslash R M S D=3.023 e-09 \backslash R M S F=1.722 e-05 \backslash D i p o l e=1.1355901$, $0.2355305,0.6684051 \backslash$

Sum of electronic and zero-point Energies= Sum of electronic and thermal Energies= Sum of electronic and thermal Enthalpies= Sum of electronic and thermal Free Energies=

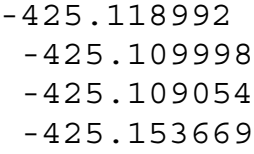

\section{Tetracyclo[3.3.0.0 $\left.{ }^{2,8} \cdot 0^{4,6}\right]$ oct-2-ene (15), NImag $=0$}

$1 \backslash 1 \backslash G I N C-N O D E 008 \backslash F O p t \backslash R B 3 L Y P \backslash 6-311+G(d, p) \backslash C 8 H 8 \backslash M I E U S S E T \backslash 13-J a n-2005 \backslash 0 \backslash$

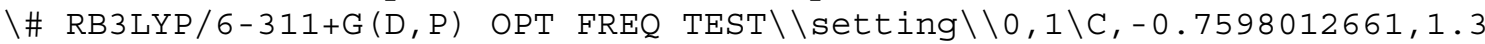


$461437549,-0.2652372936 \backslash \mathrm{C},-0.552067177,0.1478995876,-1.1993855536 \backslash \mathrm{C},-0$ $.4211512006,-1.2994513515,-0.586936291 \backslash \mathrm{C},-0.646565999,-1.1359471348,0$. $8542148836 \backslash \mathrm{C}, 0.2561040108,1.172359159,0.876376771 \backslash \mathrm{C}, 0.8026502313,-0.44$ $25439585,-0.9650380634 \backslash \mathrm{C}, 1.277026154,0.1376221027,0.3460706312 \backslash \mathrm{C}, 0.262$ $160861,-0.2552480747,1.3631158268 \backslash \mathrm{H},-1.7926836973,1.4546881733,0.07629$ $60998 \backslash \mathrm{H},-0.4970961048,2.2541979719,-0.8218169837 \backslash \mathrm{H},-0.9908435631,0.175$ $084025,-2.1885148554 \backslash \mathrm{H},-0.777955503,-2.1212505236,-1.1988888449 \backslash \mathrm{H},-1.6$ $243789284,-1.3190450139,1.2911942155 \backslash \mathrm{H}, 0.5770661198,2.0382793243,1.448$ $6936912 \backslash \mathrm{H}, 1.4729925174,-0.7320584654,-1.7626525821 \backslash \mathrm{H}, 2.3227654726,0.22$ $51000004,0.6166037931 \backslash \backslash$ Version=x86-Linux-G03RevB. $02 \backslash$ State $=1-A \backslash H F=-309$. $5735313 \backslash \mathrm{RMSD}=9.614 \mathrm{e}-09 \backslash \mathrm{RMSF}=4.041 \mathrm{e}-05 \backslash \mathrm{Dipole}=-0.2566263,0.2957867,-0.7$ $908374 \backslash \mathrm{PG}=\mathrm{CO}$ [X ( C8H8) ] \\@

Sum of electronic and zero-point Energies= Sum of electronic and thermal Energies= Sum of electronic and thermal Enthalpies= Sum of electronic and thermal Free Energies=

$$
\begin{aligned}
& -309.440875 \\
& -309.435664 \\
& -309.434719 \\
& -309.469571
\end{aligned}
$$

\section{endo-6-Ethynylbicyclo[3.1.0]hex-2-ene (17), NImag = 0}

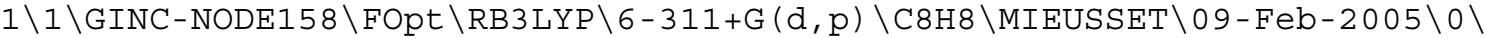

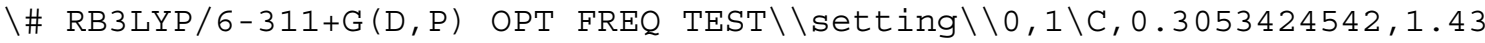
$18077973,0.7672710608 \backslash \mathrm{C},-0.3134262199,1.1448952456,-0.6007313435 \backslash \mathrm{C},-1$. $3048826252,0.0135752361,-0.4184799157 \backslash \mathrm{C},-1.2262204642,-0.3534887747,1$. $0237405113 \backslash \mathrm{C},-0.3365139706,0.4018848652,1.6731742277 \backslash \mathrm{C},-0.0347067394$, $0.200937125,-1.2602042206 \backslash \mathrm{C}, 1.9657516282,-1.7611801688,-0.5368437692 \backslash \mathrm{C}$ $, 1.0442537697,-1.0525488516,-0.8513169103 \backslash \mathrm{H}, 0.0784133805,2.4536695394$, $1.0930712481 \backslash \mathrm{H}, 1.3976307348,1.3371473082,0.757069889 \backslash \mathrm{H},-0.5230407088,1$ $.9717884109,-1.2677579356 \backslash \mathrm{H},-2.2500168717,-0.0264747146,-0.9474898518 \backslash$ $\mathrm{H},-1.7992141369,-1.1637368709,1.4573012002 \backslash \mathrm{H},-0.0589676703,0.284219147$ $8,2.713914417 \backslash \mathrm{H},-0.2156659976,-0.209726062,-2.3310984032 \backslash \mathrm{H}, 2.773274273$ $4,-2.3909361025,-0.2546684056 \backslash \backslash$ Version=x86-Linux-G03RevB .02 $\backslash$ State $=1-A \backslash$ $\mathrm{HF}=-309.6231727 \backslash \mathrm{RMSD}=7.786 \mathrm{e}-09 \backslash \mathrm{RMSF}=5.262 e-05 \backslash \mathrm{Dipole}=-0.2079471,0.3189$ $307,0.003199 \backslash \mathrm{PG}=\mathrm{CO} \quad[\mathrm{X}(\mathrm{C} 8 \mathrm{H} 8)] \backslash \backslash @$

Sum of electronic and zero-point Energies= Sum of electronic and thermal Energies= Sum of electronic and thermal Enthalpies= Sum of electronic and thermal Free Energies=

$$
\begin{aligned}
& -309.492258 \\
& -309.485454 \\
& -309.484509 \\
& -309.522987
\end{aligned}
$$

\section{$(\mathbf{1 7} \rightarrow \mathbf{1 8})$, NImag $=1$}

$1 \backslash 1 \backslash G I N C-N O D E 103 \backslash F T S \backslash R B 3 L Y P \backslash 6-311+G(d, p) \backslash C 8 H 8 \backslash M I E U S S E T \backslash 11-F e b-2005 \backslash 0 \backslash \backslash$

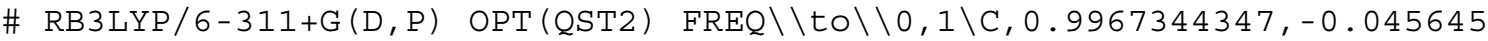
$6127,0.8685733297 \backslash \mathrm{C},-0.0144913173,1.3847640199,-0.7478481849 \backslash \mathrm{C},-1.4266$ $942985,-1.0854401124,-0.6916697089 \backslash C,-1.0483930531,0.8959237071,0.0503$ $444157 \backslash \mathrm{C}, 0.9886603496,-1.1441538517,-0.1927124959 \backslash \mathrm{C}, 1.197871604,0.8274$ $113689,-0.3406434245 \backslash \mathrm{C},-0.4145636254,0.3556379095,1.3363879519 \backslash \mathrm{C},-0.25$ $70970186,-1.5349661617,-0.5900893053 \backslash \mathrm{H}, 1.7664894599,-0.0921488308,1.63$ $53675351 \backslash \mathrm{H},-0.147554083,1.9627051886,-1.6527633686 \backslash \mathrm{H},-2.4716367925,-1$. $302341054,-0.5882664419 \backslash \mathrm{H},-2.0608917803,1.2773496936,0.0239388525 \backslash \mathrm{H}, 1$. $9184828661,-1.4692480543,-0.6358124674 \backslash \mathrm{H}, 2.1596614634,0.9964383524,-0$. $8037976132 \backslash \mathrm{H},-0.330717452,1.1685968947,2.0671672509 \backslash \mathrm{H},-0.9659961349,-0$ $.4625397915,1.8001107863 \backslash \backslash$ Version=x86-Linux-G03RevB .02 $\backslash$ State $=1-\mathrm{A} \backslash \mathrm{HF}=-3$ $09.5738612 \backslash \mathrm{RMSD}=9.397 e-09 \backslash \mathrm{RMSF}=4.958 e-05 \backslash \mathrm{Dipole}=0.1333936,0.5717657,0$.

$4001157 \backslash \mathrm{PG}=\mathrm{CO} 1 \mathrm{X}(\mathrm{C} 8 \mathrm{H} 8)] \backslash \backslash @$

Sum of electronic and zero-point Energies= Sum of electronic and thermal Energies= Sum of electronic and thermal Enthalpies= Sum of electronic and thermal Free Energies=

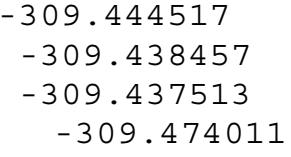


Bicyclo[3.2.1]octa-2,3,6-triene (18), NImag $=0$

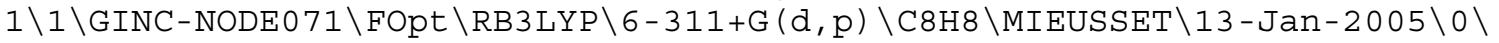

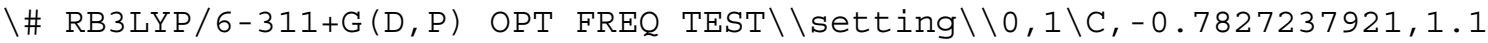
$523779452,0.0656390951 \backslash \mathrm{C}, 0.0975217675,0.6674193073,-1.101352458 \backslash \mathrm{C},-0.5$ $157790606,-0.7219394909,-1.3222696439 \backslash C,-1.0222458221,-1.1778399435,-0$ $.1645359806 \backslash \mathrm{C},-0.8563170368,-0.1624582672,0.9286919565 \backslash \mathrm{C}, 1.4763069343$, $0.1505179261,-0.5975397167 \backslash \mathrm{C}, 0.5582364018,-0.0792167482,1.6226779515 \backslash \mathrm{C}$ $, 1.5160028002,-0.0031319975,0.7205792076 \backslash \mathrm{H},-1.7750957156,1.4111951565$, $-0.313269864 \backslash \mathrm{H},-0.370666124,2.0054538416,0.6065719683 \backslash \mathrm{H}, 0.1318334006,1$ $.3319123419,-1.964238544 \backslash \mathrm{H},-0.4278473874,-1.2810292415,-2.2458590375 \backslash \mathrm{H}$ $,-1.4400098227,-2.163929726,-0.0044572154 \backslash \mathrm{H},-1.6479171434,-0.185986724$ $3,1.6781679474 \backslash \mathrm{H}, 2.108069018,-0.3787929222,-1.3036689294 \backslash \mathrm{H}, 0.595620620$ $9,0.3068048851,2.6354112057 \backslash \backslash$ Version=x86-Linux-G03RevB . 02 $\backslash$ State $=1-A \backslash H F$ $=-309.5954612 \backslash \mathrm{RMSD}=4.743 e-09 \backslash \mathrm{RMSF}=1.846 e-05 \backslash \mathrm{Dipole}=-0.7019221,0.067089$ $7,-0.3482296 \backslash \mathrm{PG}=\mathrm{CO} \quad[\mathrm{X}(\mathrm{C} 8 \mathrm{H} 8)] \backslash \backslash @$

Sum of electronic and zero-point Energies= Sum of electronic and thermal Energies= Sum of electronic and thermal Enthalpies= Sum of electronic and thermal Free Energies=

$$
\begin{aligned}
& -309.463049 \\
& -309.457325 \\
& -309.456381 \\
& -309.492173
\end{aligned}
$$

\section{endo-6-(2-Diazovinyl)bicyclo[3.1.0]hex-2-ene (26), NImag = 0}

$1 \backslash 1 \backslash G I N C-N O D E 052 \backslash F r e q \backslash R B 3 L Y P \backslash 6-311+G(d, p) \backslash C 8 H 8 N 2 \backslash M I E U S S E T \backslash 24-J a n-2006 \backslash$ $0 \backslash \backslash \# N$ GEOM=ALLCHECK GUESS=READ SCRF=CHECK TEST GENCHK RB3LYP/6-311+G (D

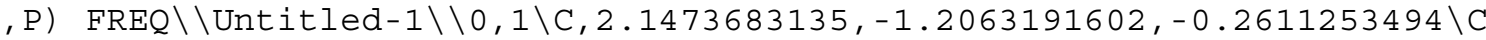
$, 1.5660281092,0.8796315081,0.756064448 \backslash \mathrm{C},-2.1391255895,0.4884842524,0$. $1473741714 \backslash \mathrm{C}, 0.2292580475,1.12796692,0.0491425881 \backslash \mathrm{C},-0.844819734,0.142$ $3202362,-0.0309568267 \backslash \mathrm{C}, 2.0008476416,-0.5474452431,1.0948407911 \backslash \mathrm{H}, 1.91$ $33002156,1.6921054315,1.3819751662 \backslash \mathrm{H},-0.1268242216,2.1466037366,0.1548$ $92724 \backslash \mathrm{H},-0.5589515163,-0.8869841726,-0.2483860353 \backslash \mathrm{H}, 2.9530393716,-0.54$ $19896533,1.6385437533 \backslash \mathrm{H}, 2.389739665,-2.2556460923,-0.3819387859 \backslash \mathrm{H}, 1.28$ $30428753,-1.0706675843,1.7372947712 \backslash \mathrm{N},-3.1017541409,-0.3981702755,0.06$ $71789283 \backslash \mathrm{N},-4.0758982193,-0.9792218607,0.047479205 \backslash \mathrm{C}, 1.5498342697,1.00$ $41716641,-0.7464370279 \backslash \mathrm{H}, 1.8778433171,1.8950891375,-1.2692327134 \backslash \mathrm{C}, 1.9$ $159214288,-0.3474162476,-1.259025919 \backslash \mathrm{H}, 1.960501894,-0.5851294291,-2.31$ $50170673 \backslash \backslash$ Version=AM64L-G03RevC.02\State $=1-\mathrm{A} \backslash \mathrm{HF}=-419.122395 \backslash \mathrm{RMSD}=1.713$

$e-09 \backslash R M S F=2.014 e-05 \backslash$ Dipole $=1.3953019,-0.1125412,-0.0224051 \backslash$

Sum of electronic and zero-point Energies=

Sum of electronic and thermal Energies=

Sum of electronic and thermal Enthalpies=

Sum of electronic and thermal Free Energies=

-418.981278
-418.972707
-418.971763
-419.015303

\section{3-Diazobicyclo[3.2.1]oct-6-en-2,3-diyl (27 singlet), NImag = 0}

$1 \backslash 1 \backslash G I N C-N O D E 107 \backslash F r e q \backslash U B 3 L Y P \backslash 6-311+G(d, p) \backslash C 8 H 8 N 2 \backslash M I E U S S E T \backslash 09-M a y-2006 \backslash$

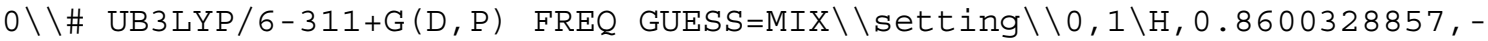
$0.2837891714,2.181779 \backslash \mathrm{C}, 0.2961388857,-0.3191931714,1.260666 \backslash \mathrm{C},-1.79263$ $81143,-0.8480441714,0 . \backslash \mathrm{C}, 0.2961388857,-0.3191931714,-1.260666 \backslash \mathrm{C},-1.192$ $6141143,-0.0585271714,-1.189293 \backslash \mathrm{C}, 0.9441058857,-0.3910351714,0 . \backslash \mathrm{C},-1.1$ $926141143,-0.0585271714,1.189293 \backslash \mathrm{H},-1.5162031143,-1.9044081714,0 . \backslash \mathrm{H},-2$ $.8812871143,-0.7559861714,0 . \backslash \mathrm{H}, 0.8600328857,-0.2837891714,-2.181779 \backslash \mathrm{H}$, $-1.6822951143,-0.2466251714,-2.145456 \backslash \mathrm{H},-1.6822951143,-0.2466251714,2$. $145456 \backslash \mathrm{C},-1.3048461143,1.3720968286,0.673844 \backslash \mathrm{H},-1.2157451143,2.2440678$ $286,1.31149 \backslash \mathrm{C},-1.3048461143,1.3720968286,-0.673844 \backslash \mathrm{H},-1.2157451143,2.2$ $440678286,-1.31149 \backslash N, 2.2930728857,-0.3629441714,0 . \backslash N, 3.4184348857,-0.3$ $891921714,0 . \backslash \backslash$ Version=AM64L-G03RevC.02\HF=-419.1116165 SS2=0.521758\S2$1=0 . \backslash S 2 A=0.018801 \backslash R M S D=3.695 e-09 \backslash R M S F=2.315 e-05 \backslash D i p o l e=0.1667481,-0.01$ $41577,0.1$

Sum of electronic and zero-point Energies= Sum of electronic and thermal Energies= Sum of electronic and thermal Enthalpies=

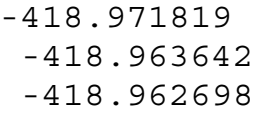




\section{(27 singlet $\rightarrow$ 28), NImag $=1$}

$1 \backslash 1 \backslash$ GINC-NODE1 $07 \backslash$ Freq \UB3LYP \6-311+G (d,p) \C8H8N2 \MIEUSSET $10-M a y-2006 \backslash$

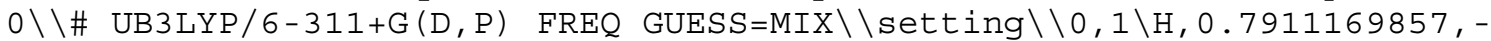
$2.1771661,-0.0053039143 \backslash \mathrm{C}, 0.2571049857,-1.2474891,-0.1412669143 \backslash \mathrm{C},-1.7$ $276400143,0.0001759,-1.1143459143 \backslash C, 0.2571959857,1.2475649,-0.14109491$ $43 \backslash \mathrm{C},-1.2292710143,1.1961019,-0.2859249143 \backslash \mathrm{C}, 0.9484209857,0.0000209,-0$ $.2145129143 \backslash \mathrm{C},-1.2293630143,-1.1960041,-0.2862669143 \backslash \mathrm{H},-1.3460770143,0$ $.0003139,-2.1379019143 \backslash \mathrm{H},-2.8207360143,0.0002019,-1.1380199143 \backslash \mathrm{H}, 0.791$ $4129857,2.1772139,-0.0057219143 \backslash \mathrm{H},-1.6959590143,2.1538229,-0.506273914$ $3 \backslash \mathrm{H},-1.6960660143,-2.1536501,-0.5069029143 \backslash \mathrm{C},-1.2418750143,-0.7034941$, $1.1324180857 \backslash \mathrm{H},-1.1990150143,-1.3429241,2.0011420857 \backslash \mathrm{C},-1.2419510143,0$ $.7031819,1.1326340857 \backslash \mathrm{H},-1.1988820143,1.3423929,2.0015100857 \backslash \mathrm{N}, 2.26728$ $59857,-0.0000521,-0.0529259143 \backslash \mathrm{N}, 3.3924959857,-0.0000271,0.0254450857 \backslash$ $\backslash$ Version=AM64L-G03RevC.02 SState $=1-\mathrm{A} \backslash \mathrm{HF}=-419.1047044 \backslash \mathrm{S} 2=0 . \backslash \mathrm{S} 2-1=0 . \backslash \mathrm{S} 2 \mathrm{~A}=$ $0 . \backslash \mathrm{RMSD}=7.580 \mathrm{e}-09 \backslash \mathrm{RMSF}=3.494 \mathrm{e}-05 \backslash \mathrm{Dipole}=-0.0068387,-0.000232,-0.093780$ $8 \backslash$

Sum of electronic and zero-point Energies= Sum of electronic and thermal Energies= Sum of electronic and thermal Enthalpies= Sum of electronic and thermal Free Energies=

-418.964588
-418.956931
-418.955987
-418.996826

\section{3-Diazobicyclo[3.2.1]oct-6-en-2,3-diyl (27 triplet), NImag $=0$}

$1 \backslash 1 \backslash G I N C-N O D E 083 \backslash F r e q \backslash U B 3 L Y P \backslash 6-311+G(d, p) \backslash$ C8H8N2 (3) \MIEUSSET \24-Jan-20 $06 \backslash 0 \backslash \backslash \# N$ GEOM=ALLCHECK GUESS=READ SCRF=CHECK TEST GENCHK UB3LYP/6-311+

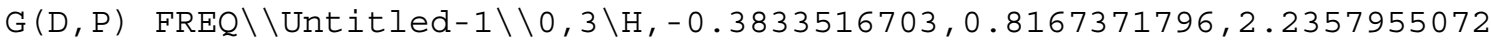
$\backslash \mathrm{C},-0.0517592574,0.3872607812,1.2981744983 \backslash \mathrm{C}, 1.7690737741,-0.750823680$ $6,0.0636691034 \backslash \mathrm{C},-0.050225718,0.434147888,-1.1349959268 \backslash \mathrm{C}, 0.7717756902$ $,-0.8359680672,-1.1214152916 \backslash \mathrm{C},-0.4141389748,0.9738466369,0.0990746972$ $\backslash \mathrm{C}, 0.7699319068,-0.8813398784,1.2429283046 \backslash \mathrm{H}, 2.3397923106,0.1795432262$ $, 0.0819347333 \backslash \mathrm{H}, 2.4544630638,-1.600633342,0.0480393929 \backslash \mathrm{H},-0.3813322474$ , $0.8934554595,-2.0581442444 \backslash \mathrm{H}, 1.2378298783,-1.0231029251,-2.0897492933$ $\backslash \mathrm{H}, 1.2330849533,-1.1046326269,2.2047445042 \backslash \mathrm{C},-0.133373518,-2.001944657$ $7,0.7056193275 \backslash \mathrm{H},-0.747427014,-2.6287765131,1.3400461679 \backslash \mathrm{C},-0.13190092$ $52,-1.9764115113,-0.6289034681 \backslash \mathrm{H},-0.7449784518,-2.5786648096,-1.287660$ $1114 \backslash \mathrm{N},-1.2423645329,2.1824645463,0.1965553767 \backslash \mathrm{N},-1.6411181369,2.81088$ $82088,-0.7136859658 \backslash \backslash$ Version=AM64L-G03RevC.02 \State $=3-\mathrm{A} \backslash \mathrm{HF}=-419.128854$ $8 \backslash S 2=2.025667 \backslash S 2-1=0 . \backslash S 2 A=2.000197 \backslash R M S D=4.591 e-09 \backslash R M S F=1.418 e-05 \backslash D i p o 1$ $e=0.7760475,-1.1768899,0.1905693$

Sum of electronic and zero-point Energies= Sum of electronic and thermal Energies= Sum of electronic and thermal Enthalpies= Sum of electronic and thermal Free Energies=

-418.988049
-418.980152
-418.979208
-419.021521

\section{3-Diazotetracyclo[3.3.0.0 $\left.{ }^{2,8} .0^{4,6}\right]$ octane (28), NImag $=0$}

$1 \backslash 1 \backslash$ GINC-NODE0 76 Freq \RB3LYP \6-311+G (d,p) \C8H8N2 \MIEUSSET $23-J a n-2006 \backslash$ $0 \backslash \backslash \# N$ GEOM=ALLCHECK GUESS=READ SCRF=CHECK TEST GENCHK RB3LYP/6-311+G(D

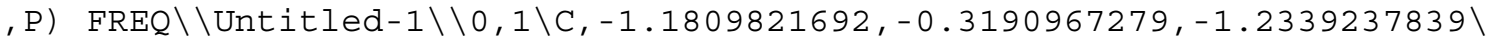
C, $-1.0112245521,1.1399956858,-0.8552491421 \backslash \mathrm{C}, 0.2114263063,0.3040261295$ $,-1.2183472253 \backslash \mathrm{C},-1.3424304164,-1.101664861,0.0573468408 \backslash \mathrm{C},-1.26800610$ $11,-0.1042755769,1.1978882894 \backslash C,-0.0470167555,-0.9484296715,0.84839166$ $12 \backslash \mathrm{C}, 0.9331800343,-0.2962292352,-0.0628239291 \backslash \mathrm{C},-1.2997476235,1.311136$ $849,0.6320435216 \backslash \mathrm{N}, 2.1861662168,-0.1465086094,0.1845955979 \backslash \mathrm{N}, 3.3150396$ $206,-0.0569579314,0.3800389029 \backslash \mathrm{H},-1.6549229148,-0.6447696378,-2.149781$ $1429 \backslash \mathrm{H},-1.2981115763,1.9260606344,-1.5447336387 \backslash \mathrm{H}, 0.7456678064,0.52892$ $10991,-2.1335555979 \backslash \mathrm{H},-1.9355546289,-2.0051432994,0.0948679809 \backslash \mathrm{H},-1.75$ 
$92969391,-0.3085965819,2.1425983452 \backslash \mathrm{H}, 0.2849774595,-1.7036462679,1.550$ $5497981 \backslash \mathrm{H},-2.2918543033,1.7515481744,0.7748702199 \backslash \mathrm{H},-0.5705381027,1.96$ $7116113,1.1207851338 \backslash \backslash$ Version=AM64L-G03RevC .02 $\backslash$ State $=1-A \backslash H F=-419.14593$ $93 \backslash \mathrm{RMSD}=2.287 e-09 \backslash \mathrm{RMSF}=4.796 e-06 \backslash \mathrm{Dipole}=-1.2167287,-0.0267888,-0.16839$ $26 \backslash$

Sum of electronic and zero-point Energies= Sum of electronic and thermal Energies= Sum of electronic and thermal Enthalpies= Sum of electronic and thermal Free Energies=

$$
\begin{array}{r}
-419.001891 \\
-418.994842 \\
-418.993897 \\
-419.033830
\end{array}
$$

\section{4+, NImag $=0$}

$1 \backslash 1 \backslash$ GINC-NODE0 48 $\backslash$ FOpt \RB3LYP \6-311+G (d,p) \C8H9 (1+) \MIEUSSET $22-$ Oct - 200 $5 \backslash 0 \backslash \backslash \#$ RB3LYP/6-311+G(D,P) OPT POP=(MINIMAL) FREQ TEST \Untitled-1\\1, $1 \backslash \mathrm{C},-0.1867899193,-0.9441102166,0.8880990823 \backslash \mathrm{C},-1.169739038,0.21418954$ $29,0.6339881697 \backslash \mathrm{C},-0.940074268,0.6488706747,-0.7171829769 \backslash \mathrm{C}, 0.18474859$ $54,-0.2515690401,-1.2690568277 \backslash \mathrm{C}, 1.173342434,0.5402913488,-0.387999485$ $4 \backslash \mathrm{C}, 0.9376454035,0.1059499769,0.9621553948 \backslash \mathrm{C},-0.0022909477,-1.57566634$ $69,-0.504871346 \backslash \mathrm{C}, 0.0037062288,1.3341630793,0.4153803577 \backslash \mathrm{H},-0.35373253$ $24,-1.5779661782,1.7512033724 \backslash \mathrm{H},-1.9758024763,0.539524267,1.2768154442$ $\backslash \mathrm{H},-1.5553431208,1.3431556147,-1.2723142506 \backslash \mathrm{H}, 0.3508810167,-0.26514298$ $65,-2.3399911104 \backslash \mathrm{H}, 1.9939913938,1.1611110412,-0.7194801175 \backslash \mathrm{H}, 1.5387763$ $371,0.3592036668,1.8243122524 \backslash \mathrm{H},-0.8830777129,-2.1303745381,-0.8343611$ $156 \backslash \mathrm{H}, 0.8767857156,-2.2208061367,-0.5602863003 \backslash \mathrm{H}, 0.0042304482,2.358581$ $1363,0.7510276148 \backslash \backslash$ Version=AM64L-G03RevC.02\State $=1-A \backslash H F=-309.962652 \backslash R$ $\mathrm{MSD}=6.943 e-09 \backslash \mathrm{RMSF}=2.577 e-05 \backslash \mathrm{Dipole}=-0.001384,0.6498647,0.2247188 \backslash \mathrm{PG}=\mathrm{C}$ $01[\mathrm{X}$ ( C8H9) ] \\@

Sum of electronic and zero-point Energies= Sum of electronic and thermal Energies= Sum of electronic and thermal Enthalpies= Sum of electronic and thermal Free Energies=

$$
\begin{aligned}
& -309.817232 \\
& -309.811652 \\
& -309.810708 \\
& -309.846441
\end{aligned}
$$

\section{$(4+\rightarrow 7+)$, NImag $=1$}

$1 \backslash 1 \backslash$ GINC-NODE $044 \backslash F T S \backslash R B 3 L Y P \backslash 6-311+G(d, p) \backslash C 8 H 9(1+) \backslash M I E U S S E T \backslash 03-N o v-2005$

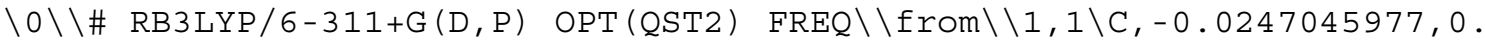
$5082818421,1.1682986789 \backslash \mathrm{C}, 1.0146324945,-0.6136969128,-0.8227518577 \backslash \mathrm{C},-$ $1.3987361022,0.027024171,-0.6725717199 \backslash \mathrm{C},-0.0244345212,0.5040761588,-1$ $.1700867768 \backslash \mathrm{C},-1.3988698817,0.0293624227,0.6722039017 \backslash \mathrm{C}, 1.0143008932,-$ $0.6106687931,0.8252004539 \backslash \mathrm{C}, 0.3317759995,1.4662124336,-0.0026403471 \backslash \mathrm{C}$, $0.437370676,-1.6094507241,0.0029228424 \backslash \mathrm{H}, 0.0382601998,0.8986768691,2.1$ $79299564 \backslash \mathrm{H}, 2.0326929587,-0.6141096093,-1.1913897209 \backslash \mathrm{H},-2.1901607153,-0$ $.331244782,-1.3180284871 \backslash \mathrm{H}, 0.038708934,0.8906620733,-2.1825470855 \backslash \mathrm{H},-2$ $.1904527487,-0.3266211732,1.3187332361 \backslash \mathrm{H}, 2.0323157361,-0.6100388732,1$. $1940053863 \backslash \mathrm{H}, 1.3528333732,1.8494914144,-0.003174312 \backslash \mathrm{H},-0.3926905934,2$. $2929887097,-0.0041842052 \backslash \mathrm{H},-0.4295169061,-2.2566482186,0.003834572 \backslash \backslash \mathrm{Ve}$ rsion=AM64L-G03RevC.02 \State $=1-\mathrm{A} \backslash \mathrm{HF}=-309.905208 \backslash \mathrm{RMSD}=7.412 \mathrm{e}-09 \backslash \mathrm{RMSF}=5$. $704 e-05 \backslash \mathrm{Dipole}=0.5366183,-0.5808451,0.0010336 \backslash \mathrm{PG}=\mathrm{C} 01$ [X(C8H9)] \\@

Sum of electronic and zero-point Energies= Sum of electronic and thermal Energies= Sum of electronic and thermal Enthalpies= Sum of electronic and thermal Free Energies=

$$
\begin{aligned}
& -309.763479 \\
& -309.757907 \\
& -309.756963 \\
& -309.792522
\end{aligned}
$$

\section{$(4+\rightarrow$ 14a+), NImag $=1$}

$1 \backslash 1 \backslash G I N C-N O D E 053 \backslash F T S \backslash R B 3 L Y P \backslash 6-311+G(d, p) \backslash C 8 H 9(1+) \backslash M I E U S S E T \backslash 25-0 c t-2005$

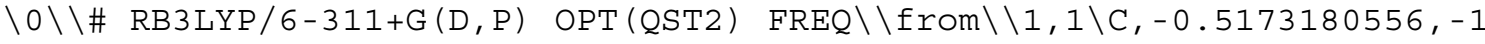
$.0918427809,0.5735918656 \backslash \mathrm{C},-1.2969549831,0.1707562021,0.2534282691 \backslash \mathrm{C},-$ $0.6319937577,0.8464218981,-0.8458559587 \backslash \mathrm{C}, 0.615257029,-0.0510101981,-1$ $.1292141416 \backslash \mathrm{C}, 1.2416317572,0.5035519597,0.1901889879 \backslash \mathrm{C}, 0.4767679612,-0$ 
$.1536518858,1.2345965652 \backslash \mathrm{C}, 0.1520424439,-1.4696445,-0.7611979585 \backslash \mathrm{C},-0$. $026132655,1.3397455889,0.4916221526 \backslash \mathrm{H},-0.9959241265,-1.8428707994,1.19$ $07938026 \backslash \mathrm{H},-2.2057957533,0.4971354276,0.7405807788 \backslash \mathrm{H},-1.1115979034,1.5$ $527861008,-1.5096720373 \backslash \mathrm{H}, 1.1567850722,0.1128081815,-2.0540990615 \backslash \mathrm{H}, 2$. $2198969457,0.9421689265,0.3325135058 \backslash \mathrm{H}, 0.6114455851,-0.0187599529,2.29$ $92289663 \backslash \mathrm{H},-0.5578744863,-1.8837417683,-1.4799419676 \backslash \mathrm{H}, 0.9824287713,-2$ $.1658605516,-0.6278851161 \backslash \mathrm{H},-0.1791625448,2.240376732,1.0655224396 \backslash \backslash \mathrm{Ve}$ rsion=AM64L-G03RevC. 02 $\backslash$ State $=1-A \backslash H F=-309.9621753 \backslash$ RMSD $=3.689 e-09 \backslash$ RMSF $=3$

$.910 e-05 \backslash \mathrm{Dipole}=-0.1879395,0.4816326,0.4973369 \backslash \mathrm{PG}=\mathrm{C} 01$ [X(C8H9)] \\@

Sum of electronic and zero-point Energies=

Sum of electronic and thermal Energies=

Sum of electronic and thermal Enthalpies=

$-309.817125$

$-309.812217$

$-309.811273$

Sum of electronic and thermal Free Energies=

$-309.845625$

\section{$(4+\rightarrow$ 18+), NImag $=1$}

$1 \backslash 1 \backslash$ GINC-NODE123 \Freq $\backslash$ RB3 LYP \6-311+G (d,p) \C8H9 (1+) \MIEUSSET \25-Jan-200

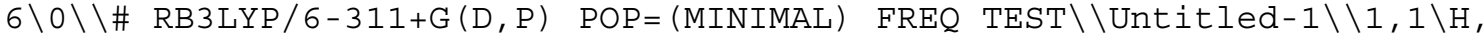
$0.7629083032,-0.4856796797,2.161377746 \backslash C, 0.4970455832,-0.2562419287,1$. $129931578 \backslash C,-0.9991014668,-0.5779690067,-0.823081066 \backslash C, 0.6435837412,1$. $2145027573,-0.67472567 \backslash C, 0.4970455832,-0.2562419287,-1.129931578 \backslash C, 0.6$ $435837412,1.2145027573,0.67472567 \backslash C,-0.9991014668,-0.5779690067,0.8230$ $81066 \backslash \mathrm{H}, 0.7629083032,-0.4856796797,-2.161377746 \backslash \mathrm{C}, 1.2607999662,-0.9777$ $260847,0 . \backslash \mathrm{H}, 1.1342191152,-2.0635373727,0 . \backslash \mathrm{H}, 2.3289067252,-0.7315896087$ $, 0 . \backslash \mathrm{C},-1.6482832048,0.3886152683,0 . \backslash \mathrm{H},-2.5663208238,0.9750371253,0 . \backslash \mathrm{H}$, $-1.5961741138,-1.1856574607,-1.501593378 \backslash \mathrm{H},-1.5961741138,-1.1856574607$ $, 1.501593378 \backslash \mathrm{H}, 0.6981458712,2.0669635853,-1.342977982 \backslash \mathrm{H}, 0.6981458712,2$ $.0669635853,1.342977982 \backslash \backslash$ Version=AM64L-G03RevC.02 $\backslash$ State $=1-A^{\prime} \backslash \mathrm{HF}=-309.9$ $115293 \backslash \mathrm{RMSD}=5.727 \mathrm{e}-09 \backslash \mathrm{RMSF}=1.051 \mathrm{e}-03 \backslash \mathrm{Dipole}=-1.0682591,0.0508387,0 . \backslash$

Sum of electronic and zero-point Energies= Sum of electronic and thermal Energies= Sum of electronic and thermal Enthalpies=

Sum of electronic and thermal Free Energies=

$-309.769185$

$-309.763718$

$-309.762774$

$-309.798169$

\section{7+, NImag $=0$}

$1 \backslash 1 \backslash$ GINC-NODE1 74 \FOpt \RB3LYP \6-311+G $(\mathrm{d}, \mathrm{p}) \backslash \mathrm{C} 8 \mathrm{H} 9(1+) \backslash$ MIEUSSET $\backslash 31-O c t-200$

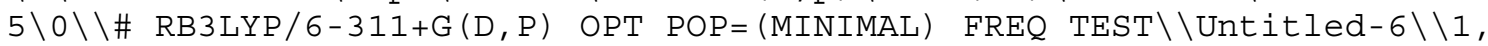
$1 \backslash C,-0.0713332158,0.4609213611,1.1905687821 \backslash C, 1.0166016538,-0.64008899$ $5,-0.9235029122 \backslash C,-1.4487407298,0.0668813252,-0.6707661991 \backslash C,-0.071063$ $3393,0.4566624147,-1.1922397828 \backslash C,-1.4488485047,0.0691175293,0.6701811$ $51 \backslash C, 1.016421201,-0.6367484074,0.9260307104 \backslash C, 0.3685911024,1.387086138$ $8,-0.0024509315 \backslash \mathrm{C}, 0.5956004007,-1.5901214739,0.00294463 \backslash \mathrm{H},-0.012182461$ $7,0.9007006889,2.1826003707 \backslash \mathrm{H}, 2.0494663629,-0.3777936109,-1.1197005199$ $\backslash \mathrm{H},-2.2756085809,-0.2077511431,-1.312157376 \backslash \mathrm{H},-0.0116511893,0.89288604$ $63,-2.1858250162 \backslash \mathrm{H},-2.2758285715,-0.2033358565,1.3123574374 \backslash \mathrm{H}, 2.049238$ $12,-0.3736984147,1.121468089 \backslash \mathrm{H}, 1.4099800927,1.7083536069,-0.0029151963$ $\backslash \mathrm{H},-0.2901476742,2.2612614302,-0.0040917544 \backslash \mathrm{H},-0.3866375073,-2.0428821$ $052,0.003671279 \backslash \backslash$ Version=AM64L-G03RevC.02 State $=1-A \backslash H F=-309.9104292 \backslash \mathrm{RM}$ $\mathrm{SD}=3.895 \mathrm{e}-09 \backslash \mathrm{RMSF}=2.882 \mathrm{e}-05 \backslash \mathrm{Dipole}=0.67673,-0.118139,0.0002927 \backslash \mathrm{PG}=\mathrm{C} 01$ $[\mathrm{X}(\mathrm{C} 8 \mathrm{H} 9)] \backslash \backslash @$

Sum of electronic and zero-point Energies= Sum of electronic and thermal Energies= Sum of electronic and thermal Enthalpies= Sum of electronic and thermal Free Energies=

$$
\begin{aligned}
& -309.766148 \\
& -309.760480 \\
& -309.759536 \\
& -309.795191
\end{aligned}
$$

\section{4a+, NImag $=0$}

$1 \backslash 1 \backslash$ GINC-NODE1 06 $\backslash F O p t \backslash R B 3 L Y P \backslash 6-311+G(d, p) \backslash C 8 H 9(1+) \backslash M I E U S S E T \backslash 22-O c t-200$

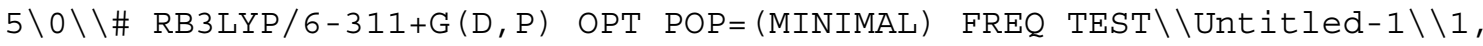


$1 \backslash \mathrm{C},-0.1911405283,-1.0446894158,0.8690578694 \backslash \mathrm{C},-1.0758245239,0.1599254$ $144,0.7287236377 \backslash \mathrm{C},-0.9593103572,0.6902957404,-0.6522562693 \backslash \mathrm{C}, 0.182132$ $076,-0.2237880961,-1.2362296399 \backslash C, 1.1779190077,0.5780980665,-0.3170723$ $39 \backslash \mathrm{C}, 0.8123952281,0.0607983883,1.0248550092 \backslash \mathrm{C}, 0.0037800811,-1.58451641$ $23,-0.5544978107 \backslash \mathrm{C}, 0.0627823279,1.4914455801,0.0989255241 \backslash \mathrm{H},-0.3590374$ $774,-1.6890899416,1.7239122198 \backslash \mathrm{H},-1.7031200883,0.5712358608,1.50749365$ $42 \backslash \mathrm{H},-1.8044693037,1.1170572754,-1.1766863902 \backslash \mathrm{H}, 0.3524183438,-0.177282$ $9374,-2.3064546631 \backslash \mathrm{H}, 2.1818050857,0.9077891985,-0.5515134084 \backslash \mathrm{H}, 1.21056$ $11759,0.4182691135,1.9644490826 \backslash \mathrm{H},-0.8714208921,-2.1296054685,-0.91341$ $23189 \backslash \mathrm{H}, 0.8862524524,-2.2218779237,-0.6377525358 \backslash \mathrm{H}, 0.0306108351,2.4380$ $8923,0.6209284706 \backslash \backslash$ Version=AM64L-G03RevC.02 \State $=1-A \backslash H F=-309.962711 \backslash R$ $\mathrm{MSD}=4.248 \mathrm{e}-09 \backslash \mathrm{RMSF}=4.094 e-05 \backslash \mathrm{Dipole}=-0.0580963,0.5255247,0.5463535 \backslash \mathrm{PG}=$

$\mathrm{CO1}[\mathrm{X}(\mathrm{C} 8 \mathrm{H} 9)] \backslash \backslash @$

Sum of electronic and zero-point Energies= Sum of electronic and thermal Energies= Sum of electronic and thermal Enthalpies= Sum of electronic and thermal Free Energies=

$$
\begin{aligned}
& -309.817314 \\
& -309.811748 \\
& -309.810804 \\
& -309.846400
\end{aligned}
$$

\section{$(\mathbf{1 4 a}+\rightarrow \mathbf{1 5}+), \mathrm{NImag}=1$}

$1 \backslash 1 \backslash G I N C-N O D E 182 \backslash F T S \backslash R B 3 L Y P \backslash 6-311+G(d, p) \backslash C 8 H 9(1+) \backslash M I E U S S E T \backslash 22-0 c t-2005$

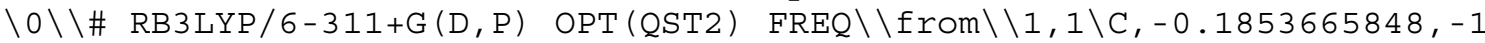
$.2025903447,0.8400365872 \backslash \mathrm{C},-0.9295483331,0.1046158913,0.899415928 \backslash \mathrm{C},-0$ $.9682876266,0.7276527669,-0.5152168867 \backslash \mathrm{C}, 0.1667056434,-0.184421215,-1$. $1800522994 \backslash \mathrm{C}, 1.1425100144,0.6215000699,-0.2009748148 \backslash \mathrm{C}, 0.6342872902,0$. $025765818,1.1320527164 \backslash \mathrm{C}, 0.0084107207,-1.5979333073,-0.5978189541 \backslash \mathrm{C}, 0$. $1316298687,1.6770139899,-0.3206215941 \backslash \mathrm{H},-0.3418102241,-1.9086240313,1$. $6489583532 \backslash \mathrm{H},-1.6090978463,0.4179902463,1.677264164 \backslash \mathrm{H},-1.9183183679,0$. $9848124635,-0.9696734453 \backslash \mathrm{H}, 0.3318334079,-0.0450620229,-2.2434328135 \backslash \mathrm{H}$, $2.2037479891,0.7785070525,-0.3570280197 \backslash \mathrm{H}, 1.0857740693,0.2821196989,2$. $0783374481 \backslash \mathrm{H},-0.8571105967,-2.1078597633,-1.0258599945 \backslash \mathrm{H}, 0.9059572309$, $-2.1973004262,-0.7640095053 \backslash \mathrm{H}, 0.1969783805,2.7657947683,-0.3854802826 \backslash$

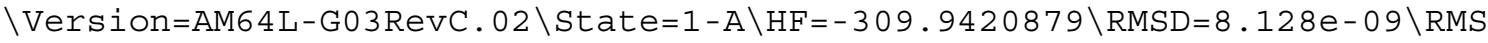
$\mathrm{F}=5.273 e-05 \backslash \mathrm{Dipole}=0.0715418,0.9798248,-0.1529796 \backslash \mathrm{PG}=\mathrm{C} 01 \quad[\mathrm{X}(\mathrm{C} 8 \mathrm{H} 9)] \backslash \backslash @$

Sum of electronic and zero-point Energies= Sum of electronic and thermal Energies= Sum of electronic and thermal Enthalpies= Sum of electronic and thermal Free Energies=

$-309.797911$

$-309.792933$

$-309.791989$

$-309.826500$

\section{4+, NImag $=1$}

$1 \backslash 1 \backslash$ GINC-NODE136\FOpt \RB3LYP \6-311+G (d,p) \C8H9 (1+) \MIEUSSET $24-$ Oct - 200 $5 \backslash 0 \backslash \backslash \#$ RB3LYP/6-311+G(D,P) OPT POP=(MINIMAL) FREQ TEST \Untitled-2\\1, $1 \backslash \mathrm{C},-0.7521065405,-1.2239894371,-0.453182716 \backslash \mathrm{C},-0.5817780094,0.0760979$ $474,-1.2061342211 \backslash \mathrm{C}, 0.7374242104,0.7503579208,-0.8470888183 \backslash \mathrm{C}, 1.035888$ $4366,-0.0984983132,0.5875442509 \backslash \mathrm{C},-0.3645248477,0.7105703,1.0892972397$ $\backslash \mathrm{C},-1.3351943419,0.0488592242,0.1178082433 \backslash \mathrm{C}, 0.5279285508,-1.517875064$ $, 0.2692432091 \backslash \mathrm{C}, 0.5594701933,1.5173436505,0.3495286533 \backslash \mathrm{H},-1.3861929131$ $,-2.0149882462,-0.8303104234 \backslash \mathrm{H},-1.049756212,0.2768281693,-2.1593767171$ $\backslash \mathrm{H}, 1.5365780435,0.9407162761,-1.5517828197 \backslash \mathrm{H}, 1.9729075201,-0.008737831$ $5,1.1226499231 \backslash \mathrm{H},-0.5652496151,0.8649024471,2.1416127819 \backslash \mathrm{H},-2.39710771$ $36,0.2280999787,0.2082496649 \backslash \mathrm{H}, 1.2723066878,-2.0325802095,-0.342481032$ $1 \backslash \mathrm{H}, 0.3915489729,-2.0644110547,1.2051557482 \backslash \mathrm{H}, 1.2623193205,2.232973099$ $1,0.7641878287 \backslash \backslash$ Version=AM64L-G03RevC.02\State $=1-A \backslash H F=-309.9676822 \backslash R M S$ $\mathrm{D}=5.281 e-09 \backslash \mathrm{RMSF}=6.365 e-05 \backslash \mathrm{Dipole}=0.6334506,0.5283364,0.3712687 \backslash \mathrm{PG}=\mathrm{C} 01$

$[\mathrm{X}(\mathrm{C} 8 \mathrm{H} 9)] \backslash \backslash @$

Sum of electronic and zero-point Energies= Sum of electronic and thermal Energies= Sum of electronic and thermal Enthalpies= Sum of electronic and thermal Free Energies=

$$
\begin{aligned}
& -309.821934 \\
& -309.816992 \\
& -309.816047 \\
& -309.850514
\end{aligned}
$$




\section{5+, NImag $=0$}

$1 \backslash 1 \backslash$ GINC-NODE0 78 \FOpt \RB3LYP \6-311+G $(\mathrm{d}, \mathrm{p}) \backslash \mathrm{C} 8 \mathrm{H} 9(1+) \backslash$ MIEUSSET $\backslash 22-$ Oct -200

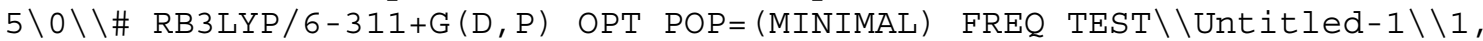
$1 \backslash \mathrm{C},-0.2003789023,-1.1958263334,0.7309488951 \backslash \mathrm{C},-0.9773155669,0.2077605$ $173,1.0857768578 \backslash C,-1.2046978307,1.0294596398,-0.0342991445 \backslash C, 0.710714$ $6151,-0.1924677144,-1.2091260745 \backslash C, 1.1468876565,0.6370397378,-0.076679$ $3856 \backslash C, 0.5492206967,-0.0199258578,1.1938636237 \backslash C, 0.0534679248,-1.45145$ $97122,-0.7253073985 \backslash \mathrm{C},-0.0675293104,1.2047822379,-0.8471986263 \backslash \mathrm{H},-0.51$ $49662897,-1.9893415683,1.3963754932 \backslash \mathrm{H},-1.6643256122,0.1322287023,1.915$ $5481917 \backslash \mathrm{H},-2.1568489312,1.5127582114,-0.232523886 \backslash \mathrm{H}, 1.1719136058,-0.13$ $75541754,-2.1866931521 \backslash \mathrm{H}, 2.0750567162,1.1932394581,-0.0591458139 \backslash \mathrm{H}, 1.0$ $304145501,0.0462205239,2.1609982248 \backslash \mathrm{H},-0.8107798745,-1.7836568494,-1.3$ $023964421 \backslash \mathrm{H}, 0.8087146137,-2.2491617585,-0.7828195526 \backslash \mathrm{H},-0.0013944746,1$ $.9590923654,-1.6172155462 \backslash \backslash$ Version=AM64L-G03RevC.02 $\backslash$ State $=1-A \backslash H F=-310$. $0018477 \backslash \mathrm{RMSD}=9.678 \mathrm{e}-09 \backslash \mathrm{RMSF}=1.163 \mathrm{e}-04 \backslash \mathrm{Dipole}=-0.240051,0.0754573,-0.06$ $80012 \backslash \mathrm{PG}=\mathrm{C} 01$ [X(C8H9) ] \\@

Sum of electronic and zero-point Energies= Sum of electronic and thermal Energies= Sum of electronic and thermal Enthalpies= $-309.856457$ $-309.850857$ $-309.849913$ Sum of electronic and thermal Free Energies= $-309.885467$

\section{$(\mathbf{1 5}+\rightarrow \mathbf{2 0}+), \mathrm{NImag}=1$}

$1 \backslash 1 \backslash G I N C-N O D E 165 \backslash F T S \backslash R B 3 L Y P \backslash 6-311+G(d, p) \backslash C 8 H 9(1+) \backslash M I E U S S E T \backslash 26-O C t-2005$

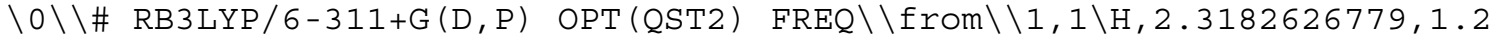
$437990514,0.3121967906 \backslash C, 1.3150904313,0.8448578185,0.252543651 \backslash C,-0.96$ $83732943,-0.0218489997,-0.9980089316 \backslash \mathrm{C}, 0.120195507,-1.3738181746,0.781$ $5524681 \backslash C,-1.1751193746,-0.6852234929,0.4030559634 \backslash C, 1.1532776949,-0.6$ $878404734,-0.1180024187 \backslash \mathrm{C}, 0.456273161,-0.2109507324,-1.2825319305 \backslash \mathrm{H},-2$ $.1284438116,-1.1227799936,0.6687691449 \backslash \mathrm{H}, 2.1306223697,-1.1431831998,-0$ $.2403523085 \backslash \mathrm{H},-1.7023960627,0.0344982,-1.7891339386 \backslash \mathrm{H}, 0.0744460471,-2$. $4335855431,0.5169383491 \backslash \mathrm{C},-1.1056596672,0.8264759667,0.2453776141 \backslash \mathrm{H},-2$ $.0289101199,1.3775570968,0.3632473593 \backslash \mathrm{H}, 0.3562923091,-1.2989067336,1.8$ $4423602 \backslash \mathrm{C}, 0.1826323127,1.4720490024,0.6147537239 \backslash \mathrm{H}, 0.1902358807,2.3982$ $877972,1.1799467002 \backslash \mathrm{H}, 0.9199900857,-0.037892164,-2.2482889549 \backslash \backslash$ Version $=A M 64 \mathrm{~L}-\mathrm{G} 03$ RevC $.02 \backslash$ State $=1-\mathrm{A} \backslash \mathrm{HF}=-309.9883829 \backslash \mathrm{RMSD}=8.616 \mathrm{e}-09 \backslash \mathrm{RMSF}=2.981 \mathrm{e}$

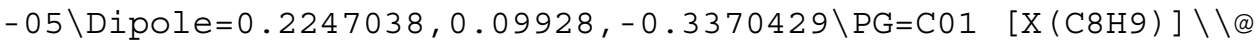

Sum of electronic and zero-point Energies=

Sum of electronic and thermal Energies=

Sum of electronic and thermal Enthalpies=

Sum of electronic and thermal Free Energies=

$-309.843570$

$-309.838329$

$-309.837385$

$-309.872390$

\section{8+, NImag $=0$}

$1 \backslash 1 \backslash$ GINC-NODE14 $0 \backslash$ FOpt $\backslash$ RB3LYP $\backslash 6-311+G(d, p) \backslash C 8 H 9(1+) \backslash M I E U S S E T \backslash 26-O c t-200$ $5 \backslash 0 \backslash \backslash \#$ RB3LYP/6-311+G(D,P) OPT POP=(MINIMAL) FREQ TEST \Untitled-6\\1, $1 \backslash C, 1.2767290885,-0.9525970266,-0.1643285126 \backslash C,-1.3501318958,0.4964157$ $6,-0.8016479504 \backslash C,-0.5393208301,-0.2427928563,1.314244321 \backslash C,-0.5902109$ $215,1.050013035,0.4533963542 \backslash C,-0.1268966971,-1.2628899777,0.201830569$ $6 \backslash C,-1.0975707695,-0.7922607811,-0.9297041263 \backslash C, 0.7839586932,1.3715238$ $365,0.0071839962 \backslash \mathrm{C}, 1.7080032823,0.3629865035,-0.2516006367 \backslash \mathrm{H}, 1.9699525$ $443,-1.7594674478,-0.3905804995 \backslash \mathrm{H},-1.9651900504,1.1338922903,-1.421288$ $548 \backslash \mathrm{H},-1.5274490647,-0.4860541327,1.7027781498 \backslash \mathrm{H}, 0.1711965947,-0.18823$ $23006,2.1411003349 \backslash \mathrm{H},-1.0866535162,1.9048651144,0.9094975192 \backslash \mathrm{H},-0.2568$ $31752,-2.3127035291,0.4590355496 \backslash \mathrm{H},-1.4813624112,-1.4844484399,-1.6666$ $826765 \backslash \mathrm{H}, 1.0699453288,2.4090655854,-0.1518081622 \backslash \mathrm{H}, 2.7190326268,0.6006$ $919006,-0.5582957581 \backslash \backslash$ Version=AM64L-G03RevC.02 \State $=1-A \backslash H F=-310.00446$ $67 \backslash \mathrm{RMSD}=4.978 \mathrm{e}-09 \backslash \mathrm{RMSF}=2.162 \mathrm{e}-06 \backslash \mathrm{Dipole}=0.664761,0.1474845,-0.0553974 \backslash$ $\mathrm{PG}=\mathrm{C} 01[\mathrm{X}$ (C8H9) ] \\@

Sum of electronic and zero-point Energies= $-309.859127$ 
Sum of electronic and thermal Energies=

Sum of electronic and thermal Enthalpies=

Sum of electronic and thermal Free Energies=
$-309.852786$

$-309.851842$

$-309.889783$

\section{$(\mathbf{1 8}+\rightarrow$ 20+), NImag $=1$}

$1 \backslash 1 \backslash$ GINC-NODE129 $\backslash$ Freq $\backslash R B 3 L Y P \backslash 6-311+G(d, p) \backslash C 8 H 9(1+) \backslash M I E U S S E T \backslash 08-M a y-200$

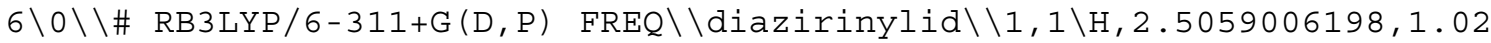
$47512066,-0.2233826225 \backslash \mathrm{C}, 1.4632644388,0.7454117486,-0.1528581125 \backslash \mathrm{C},-1$. $2143026752,-0.3136381394,-0.7971661575 \backslash \mathrm{C}, 0.2542080968,-0.9971182204,1$. $1276419485 \backslash \mathrm{C},-1.0457049122,-0.2507470274,0.8603996985 \backslash \mathrm{C}, 1.0535076598,-$ $0.7353452944,-0.1915809305 \backslash \mathrm{C},-0.0515676812,-0.8892547244,-1.2066914655$ $\backslash \mathrm{H},-1.9580099582,-0.4917500254,1.3947599515 \backslash \mathrm{H}, 1.8935565218,-1.40524571$ $44,-0.3525931265 \backslash \mathrm{H},-2.1708238082,-0.3330496254,-1.2964788815 \backslash \mathrm{H}, 0.06823$ $72438,-2.0636297064,1.2600232985 \backslash \mathrm{C},-0.9064984462,1.0125795206,0.184922$ $9625 \backslash \mathrm{H},-1.7721149432,1.6634835126,0.1295874765 \backslash \mathrm{H}, 0.7592107518,-0.61852$ $48014,2.0185018335 \backslash \mathrm{C}, 0.4541256038,1.5920233536,0.0259971665 \backslash \mathrm{H}, 0.564830$ $1518,2.6665457586,0.1087292705 \backslash \mathrm{H}, 0.0670209148,-1.4260479044,-2.1431378$ $585 \backslash \backslash$ Version=AM64L-G03RevC .02\State $=1-\mathrm{A} \backslash \mathrm{HF}=-309.9980136 \backslash \mathrm{RMSD}=3.171 \mathrm{e}-09$ $\backslash$ RMSF $=1.971 e-04 \backslash$ Dipole $=-0.4966086,-0.3635439,-0.2599441$

Sum of electronic and zero-point Energies= Sum of electronic and thermal Energies= Sum of electronic and thermal Enthalpies= Sum of electronic and thermal Free Energies=

$-309.853216$

$-309.847769$

$-309.846825$

$-309.882196$

\section{0+, NImag $=0$}

$1 \backslash 1 \backslash$ GINC-NODE145 FOpt \RB3LYP \6-311+G (d,p) \C8H9 (1+) \MIEUSSET \26-0ct-200 $5 \backslash 0 \backslash \backslash \#$ RB3LYP/6-311+G(D,P) OPT POP=(MINIMAL) FREQ TEST \Untitled-7\\1, $1 \backslash \mathrm{C}, 0.6776116969,-0.0018939563,1.5020954291 \backslash \mathrm{C}, 0.4069435309,0.045666468$ $3,-1.4257661934 \backslash \mathrm{C},-1.3413376257,-0.6949974073,0.241604293 \backslash \mathrm{C},-1.0770073$ $44,0.4356199281,-0.742789256 \backslash \mathrm{C}, 0.1083535583,-1.1311616403,0.6281694362$ $\backslash \mathrm{C}, 0.7782256923,-1.0576626169,-0.7207524759 \backslash \mathrm{C}, 0.0226085743,1.305412119$ $3,-0.4122386393 \backslash \mathrm{C}, 0.6250677234,1.2041161204,0.9462384121 \backslash \mathrm{H}, 1.036197363$ $8,-0.2137978029,2.500283963 \backslash \mathrm{H}, 0.6762006147,0.296634637,-2.4401758998 \backslash \mathrm{H}$ $,-1.8744858786,-1.513218606,-0.2436022821 \backslash \mathrm{H},-1.9302187783,-0.352167574$ $9,1.0949746608 \backslash \mathrm{H},-1.818317978,0.7376452536,-1.4740999602 \backslash \mathrm{H}, 0.175300442$ $5,-2.1077245517,1.0994539182 \backslash \mathrm{H}, 1.4540365076,-1.8208206183,-1.094739949$ $5 \backslash \mathrm{H}, 0.1324357859,2.2258402744,-0.9745290331 \backslash \mathrm{H}, 0.9460570823,2.117014897$ $7,1.4330685485 \backslash \backslash$ Version=AM64L-G03RevC.02\State $=1-A \backslash H F=-309.9980197 \backslash$ RMS $\mathrm{D}=8.725 e-09 \backslash \mathrm{RMSF}=4.311 e-05 \backslash \mathrm{Dipole}=-0.0040586,-0.1874064,-0.6575639 \backslash \mathrm{PG}=$ $\mathrm{C01}$ [X(C8H9)] \\@

Sum of electronic and zero-point Energies= Sum of electronic and thermal Energies= Sum of electronic and thermal Enthalpies= Sum of electronic and thermal Free Energies=
-309.853030
-309.846803
-309.845859
-309.883120 